\title{
Distance to the Centaurus cluster and its subcomponents from surface brightness fluctuations ${ }^{\star}$
}

\author{
S. Mieske ${ }^{1,2}$ and M. Hilker ${ }^{1}$ \\ 1 Sternwarte der Universität Bonn, Auf dem Hügel 71, 53121 Bonn, Germany \\ ${ }^{2}$ Departamento de Astronomía y Astrofísica, P. Universidad Católica, Casilla 104, Santiago 22, Chile
}

Received 19 May 2003 / Accepted 28 July 2003

\begin{abstract}
We present $I$-band Surface Brightness Fluctuations (SBF) measurements for 15 early type galaxies (3 giants, 12 dwarfs) in the central region of the Centaurus cluster, based on deep photometric data in 7 fields obtained with VLT FORS1 and with very good seeing. From the SBF-distances to our sample galaxies we determine the distance of the Centaurus cluster to be $41.3 \pm 2.1 \mathrm{Mpc}(33.08 \pm 0.11 \mathrm{mag})$. This places the Centaurus cluster at about the same distance as the "Great Attractor". We find a distance difference of $0.27 \pm 0.34$ mag between the two subcomponents Cen 30 and Cen 45, ruling out that both components are separated by their Hubble flow distance. A distance difference of $0.48 \pm 0.21$ mag is found between the central galaxies NGC 4696 (Cen 30) and NGC 4709 (Cen 45) of both components, supported by the different turn-over magnitudes of their respective globular cluster systems. This suggests that Cen 45 is falling into but has not yet reached Cen 30, supporting the idea of a large scale filament along the line of sight towards Centaurus (Churazov et al. 1999). $H_{0}=83.0 \pm 8.3 \mathrm{~km} \mathrm{~s}^{-1} \mathrm{Mpc}^{-1}$ is obtained for our Cen 30 sample taking into account the peculiar motion of the Local Group into the direction of the Centaurus cluster. This value of $H_{0}$ corresponds to a much smaller Hubble flow distortion in the direction of Centaurus than determined by Tonry et al. (2000), implying that the GA mass estimate by Tonry et al. may be too high and/or that the Centaurus cluster falls into the GA almost perpendicularly to the line of sight. As our mean single measurement error is very close to the measured distance scatter of the investigated galaxies, we can only derive an upper limit of $\pm 10 \mathrm{Mpc}$ radial extension for the Centaurus cluster, corresponding to a five times larger radial than tangential extension. No evidence for an infall pattern into the Great Attractor is found within the uncertainties for the 11 galaxies with measured redshifts.
\end{abstract}

Key words. galaxies: clusters: individual: Centaurus cluster - galaxies: distances and redshift - techniques: photometric

\section{Introduction}

\subsection{Attractors}

In the nearby universe, peculiar galaxy velocities with respect to the Hubble flow caused by clumpy matter distribution can constitute a significant fraction of the total radial velocity, which introduces a bias into the measurement of the Hubble constant $H_{0}$ if not corrected for. The two most prominent and best studied nearby matter concentrations causing deviations from the Hubble flow are the Virgo Attractor (VA) at about $17 \mathrm{Mpc}$ distance and the "Great Attractor" (GA) at about $43 \mathrm{Mpc}$ distance close to the Centaurus Cluster of galaxies (Dressler et al. 1987; Tonry et al. 2000, in the following referred to as $S B F I I$, as abbreviated by the Tonry group). The gravitational pull of the VA is about $150 \pm 50 \mathrm{~km} \mathrm{~s}^{-1}$, of the GA it is about $300 \pm 100 \mathrm{~km} \mathrm{~s}^{-1}$ (SBF II).

Send offprint requests to: $\mathrm{S}$. Mieske,

e-mail: smieske@astro.puc.cl

* Based on observations obtained at the European Southern Observatory, Chile (Observing Programme 67.A-0358).
The three-dimensional position of the VA was identified with the Virgo cluster of galaxies more than twenty years ago (e.g. Schechter 1980; Yahil et al. 1980; Tonry \& Davis 1981). Since the first postulation almost 20 years ago of a huge nearby "Great Attractor" other than the Virgo-Cluster, (e.g. Shaya 1984; Tammann \& Sandage 1984; Aaronson et al. 1986 and 1989), its approximate position, namely in direction to the Centaurus super-cluster, has not changed. However, the distance difference between the GA and the Centaurus cluster has remained uncertain. A first robust value of the GA-distance to the Local Group was derived by Lynden-Bell et al. (1988) using a projection of the Fundamental Plane (Djorgovski \& Davis 1987; Dressler et al. 1987). They derived the GA to be located at a CMB radial velocity of $4350 \pm 350 \mathrm{~km} \mathrm{~s}^{-1}$. This corresponds to $62 \mathrm{Mpc}$ for $H_{0}=70 \mathrm{~km} \mathrm{~s}^{-1} \mathrm{Mpc}^{-1}$ and is significantly behind the Centaurus cluster. Later, Tonry et al. (SBF II) refined this measurement using SBF-distances, which resulted in a somewhat closer GA distance of $43 \pm 3 \mathrm{Mpc}$. Still, this was slightly behind the position of the Centaurus cluster, which they determined to be centered at $33 \mathrm{Mpc}$. 


\subsection{The Centaurus cluster}

The proximity of the Centaurus cluster to the GA makes it an interesting subject for distance determination and to study possible effects of the GA's strong gravitational potential on the cluster's structure. If the Centaurus cluster was elongated significantly in front of and behind the GA, an infall pattern would be expected, which is an anti-correlation between redshift and distance caused by the GA's gravitational potential distorting the Hubble flow (e.g. Dressler \& Faber 1990).

An additional feature makes the Centaurus cluster even more attractive - but at the same time more complex -: in redshift space it consists of two well separated subclusters, namely the dominating component Cen 30 at about $3000 \mathrm{~km} \mathrm{~s}^{-1}$ and the 2-3 times smaller component Cen 45 at about $4500 \mathrm{~km} \mathrm{~s}^{-1}$. In several studies this remarkable substructure has been investigated (e.g. Lucey et al. 1980 $\&$ 1986; Jerjen et al. 1997; Stein et al. 1997; Churazov et al. 1999; Furusho et al. 2001), indicating that Cen 45 is probably a subgroup falling into the main cluster Cen 30 . Lucey et al. (1986) suggest that Cen 45 is located at about the same distance as Cen 30, based mainly on a comparison of the cumulative luminosity distribution in both sub-clusters. Churazov et al. (1999) propose, based on ASCA X-ray temperature measurements, that the two subcomponents are merging. They suggest the existence of a large scale filament along the line of sight towards Centaurus in order to explain the discrepancy between the unusually high velocity dispersion of the Cen 30 members and the X-ray temperature. Furusho et al. (2001) present more extended X-ray measurements and conclude that a major merger in Centaurus rather occurred several Gyrs ago. Stein et al. (1997) find that the morphological content of the two sub-clusters differs substantially. Cen 30 is more dominated by early-type galaxies, while Cen 45 contains more late-type galaxies and fewer dwarfs. This is consistent with Cen 30 being the older, main cluster, and Cen 45 the more active young infalling sub-cluster.

\subsection{Centaurus cluster galaxy distances with Surface Brightness Fluctuations}

A promising possibility to determine a precise Centaurus cluster distance and gain more insight into its spatial structure is by deriving galaxy distances using the Surface Brightness Fluctuations (SBF) method (Tonry \& Schneider 1988). The first published SBF-distances to Centaurus cluster galaxies were made by Dressler (1993), who derived distances to four Cen 30 and two Cen 45 members. He obtained a distance modulus of about $32.1 \mathrm{mag}$ for Cen 30 and $32.2 \mathrm{mag}$ for Cen 45, yielding high peculiar velocities of about $1400 \mathrm{~km} \mathrm{~s}^{-1}$ for the investigated galaxies.

Later, these measurements were refined and complemented by Tonry et al. (Tonry et al. 1997; and Tonry et al. 2001, in the following $S B F I V$ ) in the course of their SBF survey, resulting in a somewhat greater distance for Centaurus. They obtained distance moduli to 5 Cen 30 and 3 Cen 45 members. The resulting mean distance moduli are $32.51 \pm 0.11 \mathrm{mag}$ for Cen 30 and $32.80 \pm 0.09$ mag for Cen 45 , showing a distance difference between the two sub-clusters at $1.45 \sigma$ significance. However, already in SBF IV and Blakeslee et al. (2002) it has been pointed out that these results are subject to a selection effect biasing towards closer distances by up to 0.3 mag: the sensitivity of Tonry's survey is reached at about the distance of the Centaurus cluster. This makes those galaxies whose observational and statistical errors place them closer than the mean cluster distance more probable to be included in their survey than those who fall behind the cluster for their errors. A discussion of this will be given in Sect. 5 of this paper.

Deeper and more numerous SBF measurements than those of Tonry et al. are needed in order to reduce this selection effect and allow a less biased calculation of the Centaurus cluster distance.

\subsection{Aim of this paper}

To improve the distance precision to the entire Centaurus cluster and its subcomponents, we present in this paper new SBF distance measurements to 15 early type Centaurus galaxies -3 giants and 12 dwarfs. The data originate from deep VLT FORS 1 exposures in the $I$-band of six different $7 \times 7^{\prime}$ fields in the central Centaurus cluster. Of the 15 galaxies, 11 have measured radial velocities. 8 of them belong to Cen 30 and 3 to Cen 45. The two major giant elliptical galaxies of Cen 30 and Cen 45, namely NGC 4696 and NGC 4709, constitute the overlap between Tonry's and our dataset.

SBF have been measured for only small samples of dwarf galaxies, yet (e.g. Bothun 1991; Jerjen et al. 1998, 2000, 2001 and Jerjen 2003). Recently, Mieske et al. (2003, in the following MieskeI) have presented SBF simulations to test the potential of the SBF-Method to measure distances to dwarf galaxies. To our knowledge, the sample presented in this paper is the largest homogeneous sample of dwarf galaxies with SBF-distances up to now.

The paper is structured as follows: Sect. 2 explains how the absolute fluctuation magnitude $\bar{M}_{I}$ is derived from $(V-I)$. In Sect. 3, the data and their reduction are described. Section 4 shows the results of the SBF measurements. They are discussed in Sect. 5. We finish this paper with the conclusions in Sect. 6.

\section{Deriving $\bar{M}_{I}$ from $(V-I)$}

The observable derived from SBF measurements is the apparent fluctuation magnitude $\bar{m}$, equivalent to the luminosity weighted mean apparent luminosity of the stellar population. To estimate the distance of a galaxy with the SBF-Method, the absolute fluctuation magnitude $\bar{M}$ must be derived from a distance independent observable.

Tonry et al. (1997, in the following SBF I; and SBF IV) have established an empirical calibration between colour $(V-I)$ and the absolute fluctuation magnitude $\bar{M}_{I}$ :

$\bar{M}_{I}=-1.74+4.5 \times((V-I)-1.15) \mathrm{mag}$.

According to Tonry et al. (SBF IV), this equation is valid only for galaxies with $(V-I)>1.0$. As the galaxies investigated in 
this paper span a somewhat larger range of colour, $0.84<(V-$ $I)<1.35$ mag (see Table 2), it must be investigated whether for the three galaxies in our sample with $(V-I)<1.0 \mathrm{mag}$, Eq. (1) can be applied.

There is currently no published data available for $I$-band SBF measurements for $(V-I)<1.0$ mag. However, Jerjen et al. $(1998,2000)$ conclude from $R$-band SBF measurements of nearby dEs that for blue $(B-R)$ the relation between $\bar{M}_{R}$ and $(B-R)$ bifurcates into a steep one continuing the relation found for red dEs and a shallower one, giving fainter $\bar{M}_{R}$ at a given $(B-R)$.

To look into this in more detail, in Fig. 1 theoretical values of $\bar{M}_{I}$ vs. $(V-I)$ are plotted for a range of metallicities and ages typical for early type galaxies, using models of Worthey (1994) and Liu et al. (2000). For red colours, both models trace Eq. (1) well, with the Worthey-models being more deviant from the empirical calibration than the Liu-models. In the blue range, the Worthey-models for 8 and 12 Gyrs trace Eq. (1), while the 17 Gyr Worthey-models and the metal-poor Liu-models predict $\bar{M}_{I}$ substantially fainter than according to Eq. (1). This is in agreement with Jerjen's findings and shows that a correction of Eq. (1) towards fainter $\bar{M}_{I}$ should be applied for blue colours. To this end, we follow the same line of argument made in MieskeI, such that for $(V-I)<1.0$ we adopt the average of $\bar{M}_{I}$ according to Eq. (1) and a constant value of $\bar{M}_{I}=-2.4 \mathrm{mag}$, which is $\bar{M}_{I}$ at $(V-I)=1.0$. This yields the following equation holding for $(V-I)<1.0$ :

$\bar{M}_{\mathrm{I}}=-2.07+2.25 \times((V-I)-1.15) \mathrm{mag}$.

In Fig. 1, this is indicated graphically. For $(V-I) \geq 1.0$, Eq. (1) is adopted.

Note that the adjustment for $(V-I)<1.0$ should serve to decrease possible systematic biases occurring when applying an unchanged Eq. (1), but it should not be considered especially accurate. The lack of observational data in this colour regime and the discrepancy between different model grids forces us to adopt a "best guess". After all it is worth remarking that the changes discussed only apply to 3 of the 15 sample galaxies and are of the order of 0.3 mag or smaller.

It is necessary to define the intrinsic uncertainty of the two above equations, also referred to as cosmic scatter. The cosmic scatter of Eq. (1) has already been determined by Tonry et al. in $S B F I V$ to be between 0.05 and $0.10 \mathrm{mag}$. They base this finding on the scatter of their SBF distance measurements for galaxies with known distances. Tonry et al. investigated only giant galaxies. As we investigate giants and dwarfs, we do not expect a smaller spread in stellar contents than if investigating only giants. We therefore adopt the upper limit of $0.10 \mathrm{mag}$ as the cosmic scatter for $(V-I) \geq 1.0$. For $(V-I) \leq 1.0$, we adopt the quadratic sum of the former 0.10 mag scatter and the magnitude difference between Eq. (2) and Eq. (1). This amounts up to 0.37 mag for the bluest of our investigated galaxies $((V-I)=$ $0.84)$.

\section{The data}

The data for this publication have been obtained in service mode at the Very Large Telescope (VLT) of

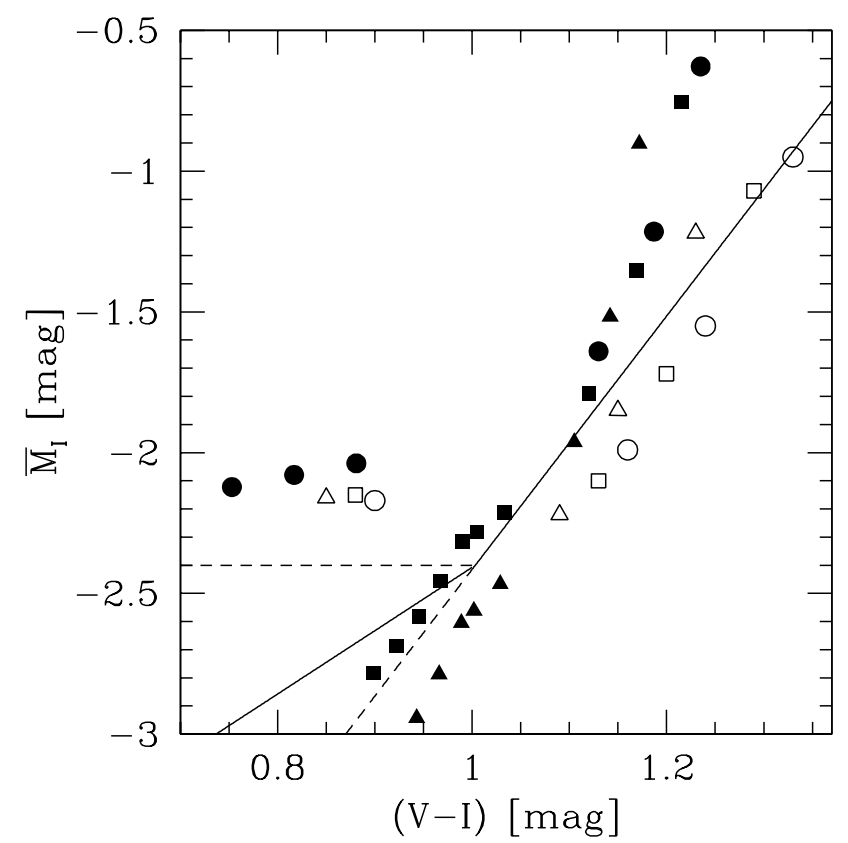

Fig. 1. Theoretical values of $\bar{M}_{I}$ plotted vs. $(V-I)$ for a range of metallicities $(-1.7$ to 0 dex $)$ and ages $(8,12$ and $17 \mathrm{Gyr})$, taken from Liu et al. (2000) (open symbols) and Worthey (1994) (filled symbols). The different symbols represent different ages as follows: triangle 8 Gyrs, square 12 Gyrs, circle 17 Gyrs. For the 17 Gyr Worthey-models, the lowest metallicity is -1.3 dex. Metallicity increases towards redder $(V-I)$ and fainter $\bar{M}_{I}$. The solid line represents the $\bar{M}_{I}-(V-I)$ relation adopted in this paper. The dashed lines for $(V-I) \leq 1.0$ indicate the constant $\bar{M}_{I}=-2.40 \mathrm{mag}$ and Eq. (1)'s continuation (see text for further details).

the European Southern Observatory, Chile (Observing Programme 67.A-0358), using UT 1 with the instrument FORS1 in imaging mode. Seven $7 \times 7^{\prime}$ fields in the central Centaurus cluster have been observed in Johnson $V$ and $I$ pass-bands. The seeing ranged between 0.4 and $0.6^{\prime \prime}$. The total integration time was $1500 \mathrm{~s}$ for the $V$ exposures, divided up into 4 dithered single exposures, and $3000 \mathrm{~s}$ for the $I$ exposures, divided up into 9 dithered single exposures. Figure 2 shows a map of the central Centaurus cluster with the observed fields and cluster galaxies indicated. Table 1 gives the coordinates of the observed fields. Table 2 gives the photometric properties and coordinates of the 15 investigated cluster galaxies. They span a magnitude range of $19.6>V>11.5 \mathrm{mag}$, corresponding to approximately $-21.5<M_{V}<-13.5$.

In the 7 fields, there are located 14 additional galaxies cataloged as early-types in the CCC. These could not be investigated for the following reasons: 5 galaxies showed pronounced spiral features on our high resolution images, revealing that they are probably late-type background galaxies rather than early-type cluster members; 6 galaxies were too faint to detect a significant SBF signal; 2 galaxies showed pronounced boxy residuals after subtracting an elliptical light model, with the boxy features having a scale size of only a few times that of the seeing; 1 galaxy was too close to the halo of a bright saturated star to obtain a reliable SBF signal. 
Table 1. Central coordinates and photometric calibration coefficients for the 7 VLT FORS1 fields as indicated in Fig. 2.

\begin{tabular}{lllllrlll}
\hline \hline Field & RA [2000] & Dec [2000] & \multicolumn{1}{l}{$Z P_{I}$} & \multicolumn{1}{l}{$Z P_{V}$} & $C T_{I}$ & $C T_{V}$ & $k_{I}$ & $k_{V}$ \\
\hline 1 & $12: 48: 45.0$ & $-41: 18: 20$ & 26.582 & 27.472 & 0.00 & 0.08 & 0.093 & 0.145 \\
2 & $12: 49: 18.5$ & $-41: 18: 20$ & 26.60 & 27.446 & 0.00 & 0.08 & 0.093 & 0.145 \\
3 & $12: 49: 52.0$ & $-41: 21: 02$ & 26.672 & 27.514 & -0.088 & 0.015 & 0.093 & 0.145 \\
4 & $12: 49: 52.0$ & $-41: 14: 50$ & 26.588 & 27.446 & 0.00 & 0.08 & 0.093 & 0.145 \\
5 & $12: 48: 45.0$ & $-41: 24: 32$ & 26.548 & 27.446 & 0.00 & 0.08 & 0.093 & 0.145 \\
6 & $12: 48: 45.0$ & $-41: 30: 44$ & 26.548 & 27.446 & 0.00 & 0.08 & 0.093 & 0.145 \\
7 & $12: 48: 45.0$ & $-41: 36: 56$ & 26.582 & 27.472 & 0.00 & 0.08 & 0.093 & 0.145 \\
\hline
\end{tabular}

Table 2. Coordinates and photometric properties of the investigated galaxies. The galaxies are ordered by field-number, and within the same field by right ascension. The field number refers to the fields indicated in Fig. 2. The radial velocities are taken from Stein et al.'s (1997) catalog of radial velocities for the Centaurus cluster.

\begin{tabular}{llllllll}
\hline \hline CCC-Nr. $^{*}$ & Field & RA $^{*}[2000]$ & Dec $^{*}[2000]$ & $V_{0}^{* *}[\mathrm{mag}]$ & $(V-I)_{0}{ }^{* *}[\mathrm{mag}]$ & $v_{\text {rad }}\left[\mathrm{km} \mathrm{s}^{-1}\right]$ & Type $^{*}$ \\
\hline 52 & 1 & $12: 45: 44.3$ & $-41: 02: 58$ & 17.86 & 1.09 & - & $\mathrm{dE}, \mathrm{N}$ \\
61 & 1 & $12: 48: 39.7$ & $-41: 16: 05$ & 16.26 & 1.14 & 2910 & $\mathrm{dE}, \mathrm{N}$ \\
65 (NGC 4696) & 1 & $12: 48: 49.0$ & $-41: 18: 39$ & 11.50 & 1.24 & 2985 & E4,S03(4) \\
70 & 1 & $12: 48: 53.9$ & $-41: 19: 09$ & 16.69 & 1.24 & 2317 & cdE (E0 in CCC) \\
75 & 1 & $12: 49: 01.9$ & $-41: 15: 36$ & 17.31 & 1.12 & 1958 & $\mathrm{dE}, \mathrm{N}$ \\
89 & 2 & $12: 49: 18.2$ & $-41: 20: 07$ & 15.43 & 1.15 & 3104 & E1 \\
111 & 3 & $12: 49: 40.0$ & $-41: 21: 59$ & 15.86 & 1.01 & 2880 & $\mathrm{dE}, \mathrm{N}$ \\
115 & 3 & $12: 49: 46.5$ & $-41: 22: 08$ & 18.15 & 0.99 & - & $\mathrm{dE}$ \\
121 & 3 & $12: 49: 54.2$ & $-41: 20: 24$ & 17.36 & 1.07 & 4739 & $\mathrm{dE}(\mathrm{Im}$ in CCC) \\
123 & 3 & $12: 49: 56.1$ & $-41: 24: 04$ & 17.35 & 1.03 & 4661 & $\mathrm{dS0}$ \\
124 & 3 & $12: 49: 56.2$ & $-41: 23: 22$ & 19.09 & 0.84 & - & $\mathrm{dE}$ \\
130 (NGC 4709) & 3 & $12: 50: 04.0$ & $-41: 22: 57$ & 12.5 & 1.35 & 4650 & $\mathrm{E} 3$ \\
125 & 4 & $12: 49: 56.4$ & $-41: 15: 37$ & 16.06 & 1.08 & 2880 & $\mathrm{dE}, \mathrm{N}$ \\
58 & 5 & $12: 48: 36.1$ & $-41: 26: 25$ & 16.78 & 1.02 & 3304 & $\mathrm{dE}$ \\
68 & 6 & $12: 48: 52.5$ & $-41: 32: 25$ & 19.63 & 0.93 & - & $\mathrm{dE}$ \\
\hline
\end{tabular}

* As in the Centaurus cluster Catalog (CCC, Jerjen et al. 1997). Note that galaxy CCC 121 is cataloged in the CCC as being of type Im. Based on our high resolution photometry, we cannot confirm this morphological type but rather find it has a normal, smooth dE-like morphology. Galaxy CCC 70 is cataloged as type E0 due to its relatively high surface brightness. However, it has $M_{V} \simeq-16.5$ mag, placing it into the dwarf galaxy regime. We therefore adopt the type compact $\mathrm{dE}$ (cdE) for CCC 70.

${ }^{* *}$ Based on this paper.

\subsection{Data reduction before SBF measurement}

The pipeline reduced images still showed large scale sky count variations of the order of $\pm 3 \%$. To partially compensate for that, a master flat field was constructed by combining all single exposures from all fields, disregarding contribution from astronomical objects to the final master flat using a sigma-clippingrejection. After division by the smoothed master flat field, the large scale variations were reduced to $\pm 1 \%$.

The observational zero points were obtained separately for each night. For all fields except for field 3, which was taken two months after the rest of the images, the colour terms were identical to within their errors. For all fields, the extinction coefficients were identical to within their errors. The accuracy of the derived zero points was of the order of $1 \%$. Table 1 gives the calibration coefficients and central coordinates of all 7 Centaurus fields.

For each field and passband, the single exposures were brought into a common coordinate system by applying integer pixel shift corrections between the single dithered frames. For SBF measurements, only integer pixel shifts are suitable, as otherwise correlated noise would be introduced. Cosmic rays were removed from the single frames using the IRAF task COSMICRAYS. The registered cleaned single frames were averaged using an average sigma clipping algorithm.

For each investigated galaxy, the local background level was determined in both pass-bands via a curve of growth analysis, yielding the total apparent magnitudes in $V$ and $I$, a surface brightness profile and a colour map. To correct for galactic reddening and absorption, we used the values from Schlegel et al. (1998), who give $A_{I}=0.221$ and $E(V-I)=0.157$ for the coordinates of the Centaurus cluster.

\subsection{SBF measurement}

The aim of the SBF measurement is the determination of the apparent fluctuation magnitude $\bar{m}_{I}$. From $(V-I)_{0}$ one then derives $\bar{M}_{I}$ via Eqs. (1) and (2) and thereby distance modulus. The following steps have been performed to measure $\bar{m}_{I}$, see as well MieskeI:

1. Model mean galaxy light with ELLIPSE using a sigma 


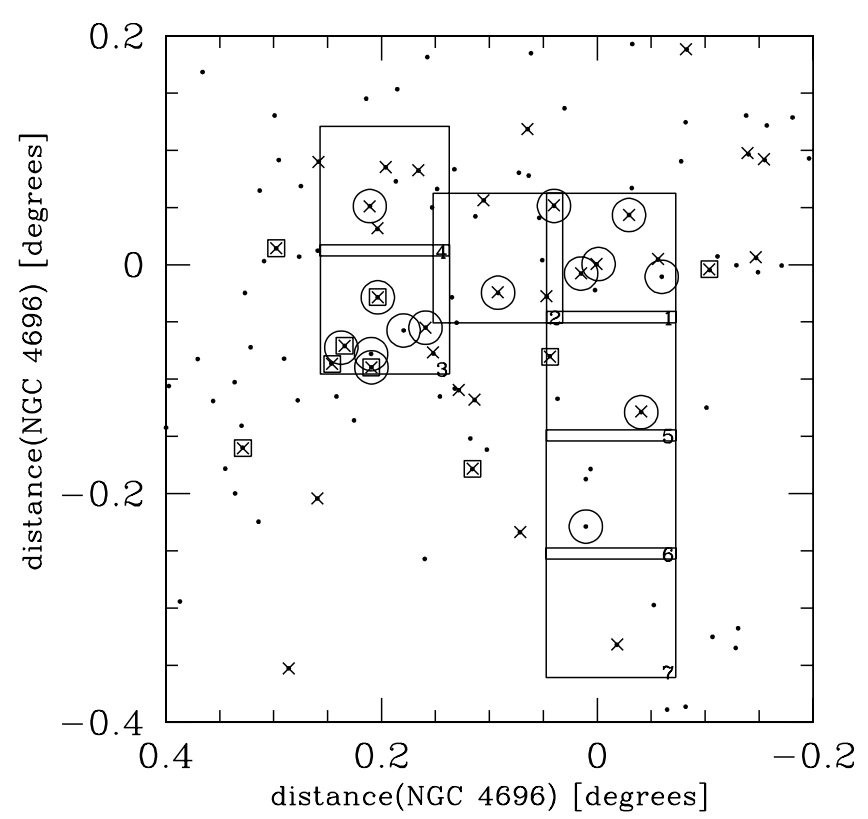

Fig. 2. Map of the central Centaurus cluster, with distances relative to the main galaxy of Cen 30, NGC 4696. East is left, North is up. Large squares are the observed VLT fields, the field number is indicated in the lower right corner of each square. Dots represent the galaxies listed in Jerjen et al.'s (1997) Centaurus Cluster Catalog (CCC) as probable and likely cluster members. Dots marked with crosses are galaxies for which Stein et al. (1997) have obtained radial velocity. Small squares indicate galaxies belonging to the sub-cluster Cen 45 . Galaxies marked with large circles are the ones for which we present new SBF measurements in this paper.

clipping algorithm to disregard contaminating sources hidden below the galaxy light, subtract the model.

2. Detect and subtract remaining contaminating objects from original image.

3. Model mean galaxy light on the cleaned image.

4. Subtract model of original image.

5. Divide resulting image by square root of the model, cut out circular portion with radius typically 20 pixel ( 4 "), corresponding to about 8 seeing disk diameters.

6. Mask out contaminating sources like foreground stars, background galaxies and globular clusters. The completeness limit of the contaminating source detections was determined by artificial star experiments using SExtractor and the ARTDATA package under IRAF. The limiting magnitude for point sources was about 25 mag in $I$.

7. Calculate the power spectrum (PS) of the cleaned image.

8. Obtain the azimuthal average of the PS.

9. Fit function of the form

$P(k)=P S F(k) \times P_{0}+P_{1}$

to the azimuthally averaged PS. PS F $(k)$ is the PS of the seeing profile, normalized to unity at $k=0$. It is determined from a single star with no close neighbours in the same frame by fitting a Moffat profile to its PS. $P_{1}$ is the white noise component. $P_{0}$ is the amplitude of the pixel-to-pixel surface brightness fluctuations. We define as the signal-to-noise of the measurement $S / N \equiv P_{0} / P_{1}$. Values at small $k$ (long wavelength) are rejected for the fit, as they are often influenced by large scale residuals from imperfect galaxy subtraction (for more details see MieskeI).

10. It holds for the desired observable $\bar{m}_{I}$ :

$\bar{m}_{\mathrm{I}}=-2.5 * \log \left(P_{0}\right)+Z P-A_{I}-\Delta k+\Delta G C-\Delta \operatorname{sim}$

with $Z P$ being the photometric zero point including exposure time. $A_{I}$ is the foreground absorption, $\Delta k=z \times 7$ the k-correction for SBF in the $I$-band (Jensen et al. 1998), $\triangle G C$ the contribution to the fluctuations caused by Globular Clusters (GCs) below the detection limit and $\Delta \operatorname{sim}=0.15 \pm$ 0.05 mag the bias correction that needs to be applied following the results of our SBF-simulations from MieskeI. Before going further, we treat in some more detail the two corrections $\Delta \operatorname{sim}$ and $\Delta G C$. As already shown in MieskeI, the fluctuations caused by background galaxies are negligible at the given depth of our data.

In Fig. 3 thumbnail images of 4 investigated galaxies are given, illustrating the reduction procedure until obtaining the azimuthally averaged power spectrum.

\subsubsection{Including the bias found in simulations}

In MieskeI we have presented SBF-simulations for artificial dEs at distances between 29.4 and 33.4 mag, including varying seeing and integration times. The simulations are based on VLT-FORS1 zero points. Their main purpose was to establish realistic magnitude limits down to which the SBF-method can be applied in order to establish the membership of a candidate $\mathrm{dE}$ in a galaxy cluster, depending on seeing and integration times.

A useful byproduct of these simulations are the error estimates for measuring $P_{0}$, and bias estimates between input and output $P_{0}$. In MieskeI we find a systematic bias of $\Delta$ sim $=$ $0.15 \pm 0.05$ mag between input and output SBF-magnitude towards measuring too faint SBF, which is independent on the simulated galaxy distance. We show that the implementation and measurement of not seeing convolved pixel-to-pixel SBF yields no bias at all and that convolution with the seeing yields less than $1 \%$ flux loss. From that it is deduced that recovering pixel-to-pixel fluctuations from seeing convolved images is subject to small, but non negligible loss of fluctuation signal in our simulations. We use the same SBF-measurement procedure both for our real data presented here and for the simulations in MieskeI. Therefore, we apply the bias estimates found in the simulations to our results.

\subsubsection{Contributions from Globular Clusters}

To calculate $\Delta G C$, Eq. (10) from Jensen et al. (1998) is used. $-8.5 \mathrm{mag}$ is assumed as the absolute $I$ band turnover magnitude TOM (Kundu \& Whitmore 2001) of the $G C$ luminosity function. Apart from the approximate distance of the investigated galaxy, the most important ingredient of Jensen's Eq. (10) is the specific frequency $S_{N}$ of $G C \mathrm{~s}$, defined as the number of $G C$ s per $M_{V}=-15$ mag galaxy luminosity. For all investigated galaxies except for the giants NGC 4696 and NGC 4709, 

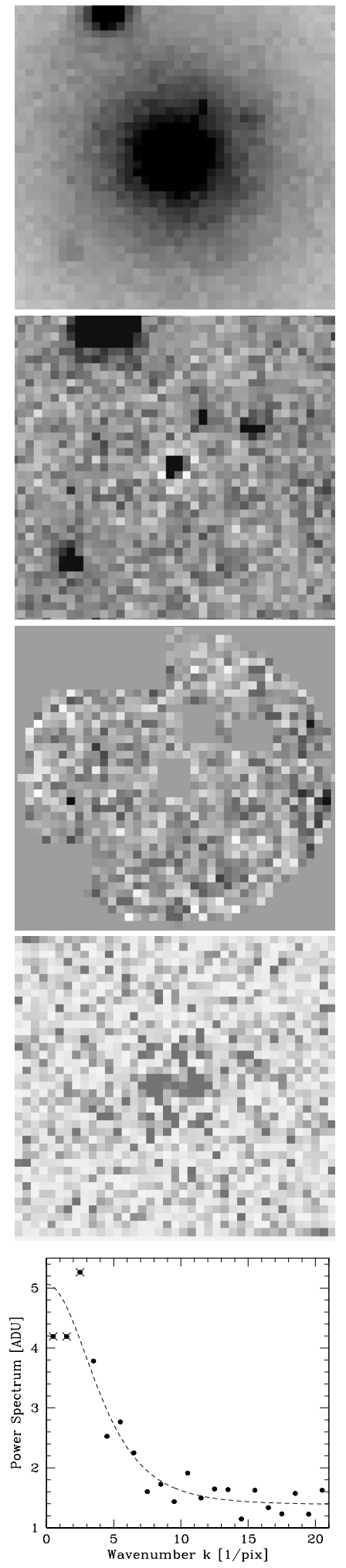

Fig. 3. Example images and plots for four galaxies, illustrating the SBF measurement procedure. From left to right: CCC 115 (field 3 ); CCC 75 (field 1); CCC 61 (field 1); CCC 125 (field 4). From top to bottom: Original galaxy image; elliptical model subtracted from former image; former image divided by square root of model, with contaminating sources and region outside measurable circle masked; power spectrum of the former image; azimuthal average of the former image with dashed line showing the result of the fit and crosses marking the points rejected for the measurement.

$S_{N}$ could not be measured precisely due to the low number counts involved: first, the expected total number of $G C$ s $N_{G C}$
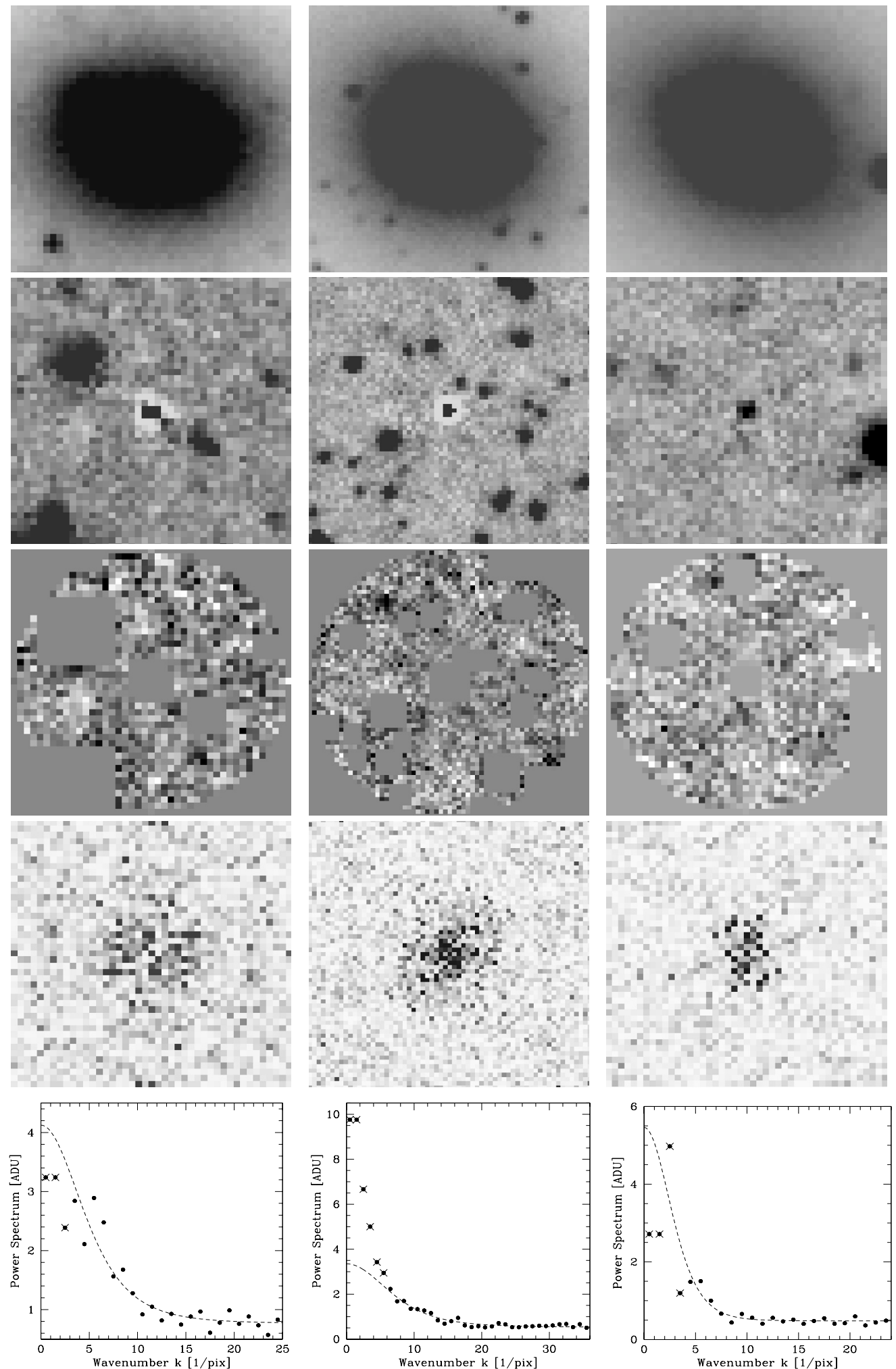
detected due to the completeness limit. Therefore, we use the results of Miller et al. (1998) on $S_{N}$ for dwarf elliptical galaxies and adopt a value of $S_{N}=4 \pm 3$ for all investigated dwarf galaxies plus the fainter giant CCC 89. The error in $\triangle G C$ is given by the error range of \pm 3 for $S_{N}$. As at a given $S_{N}$, the distance $(m-M)$ of the galaxy must be known to correctly calculate $\triangle G C, \triangle G C$ and $(m-M)$ were determined iteratively. $\triangle G C$ for the different galaxies ranged between 0.02 and $0.28 \mathrm{mag}$ with a mean of $0.11 \mathrm{mag}$. In general, $\triangle G C$ is larger for redder than for bluer galaxies, as the strength of the SBF decreases with redder colour while the assumed GC contribution remains equal.

\subsubsection{Error calculation for $(m-M)$}

The error of $(m-M)$ consists of the measurement error $\delta \bar{m}_{I}$ and the derivation error $\delta \bar{M}_{I}$.

$\delta \bar{m}_{I}$ is composed by the single errors of the different terms from Eq. (4). The most important error contribution here comes from $P_{0}$. We derive the error of $P_{0}$ from Monte Carlo simulations, using the scatter of $P_{0}$ measurements on simulated dEs from Mieske I, complemented for fainter magnitudes by new simulations. In Fig. 4, apparent magnitude $V_{0}$ is plotted vs. SBF measurement deviation $\delta P_{0}$ for 108 simulated dEs at the approximate Centaurus cluster distance. The magnitude range $15.1<V_{0}<19.6$ was chosen to cover the same range occupied by the real galaxies in this paper, excluding the magnitude regime of the two giants NGC 4709 and NGC 4696 (see Sect. 3.2.4). We subdivide the simulated magnitude range in three bins of 1.5 mag width. Within each bin, both the mean $\delta P_{0}$ and the rms-scatter around it is indicated in Fig. 4. The scatter is 0.26 mag for the brightest bin and 0.42 mag for both fainter bins. We adopt these rms-scatters in the different bins as the error in $P_{0}$ for our measurements. The corresponding mean $\mathrm{S} / \mathrm{N}$ of the $\mathrm{SBF}-$ measurement ranges between 3 for the faintest bin and 9 for the brightest one. We apparently do not deal with high $\mathrm{S} / \mathrm{N}$ data nor with large sample areas for SBF measurement, which explains the considerable uncertainty in the fainter magnitude bins, corresponding to about $20 \%$ in distance. The slightly larger mean for the brightest bin is caused by the stronger contribution from undetected GCs to the SBF-signal, as the brighter the galaxies the redder the colour and the weaker the SBF-signal. Note that the means of $\delta P_{0}$ agree with the adopted $0.15 \mathrm{mag}$ bias correction (see Sect. 3.2.1).

$\delta \bar{M}_{I}$ is composed by the cosmic scatter of $0.10 \mathrm{mag}$ and the error in measuring $(V-I)$ multiplied by the slope of Eqs. (1) or (2), respectively.

\subsubsection{Special treatment for NGC 4696 and NGC 4709}

The SBF measurement procedure was slightly different for the two bright and extended giants NGC 4696 and NGC 4709.

The first difference was that SBF were measured in adjacent rings centered on the galaxy, not in circles, leaving out the central part. This was necessary, because in their innermost part the Poisson noise caused by the high surface

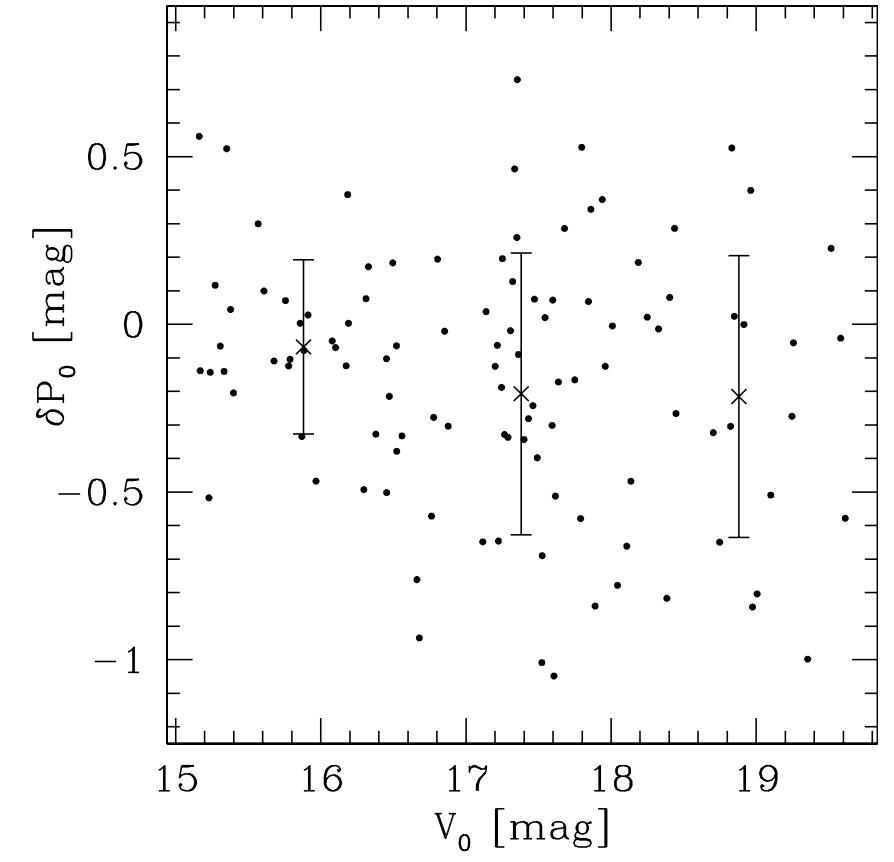

Fig. 4. Apparent magnitude $V_{0}$ of simulated dEs at 32.8 mag distance modulus (taken from Fig. 8 of Mieske $I$ for $V_{0}<17.7$ mag, new simulations otherwise) plotted vs. the measurement deviation $\delta P_{0}$ between simulated and measured SBF-magnitude. The $y$-positions of the three error bars indicate the mean $\delta P_{0}$ in the range \pm 0.75 mag of their $x$-position. The size of the error bars corresponds to the rms-scatter around the mean $\delta P_{0}$ in that magnitude range.

brightness $\left(\mu_{I} \simeq 18 \mathrm{mag} / \operatorname{arcsec}^{2}\right)$ gives a considerable shift to the bright of the completeness magnitude for detecting $G C \mathrm{~s}$, resulting in a large uncertainty when calculating the contribution from undetected $G C$ s. To avoid that, the inner galaxy parts with intensity at least half that of the sky were disregarded for the SBF measurement (see as well Tonry \& Schneider 1988). Besides, NGC 4696 has prominent dust lanes close to its center which do not allow SBF measurement. The final value of $P_{0}$ for NGC 4696 and NGC 4709 corresponds to the mean value obtained in the different rings after scaling $P_{0}$ according to the difference between $(V-I)$ in the respective ring and the mean $(V-I)$ of all rings. This is done as a consequence of the colour$\mathrm{SBF}$ relation. The error is adopted as the standard deviation of the different values. For NGC 4696, the SBF were measured in 3 rings ranging between 52 and 100" from the galaxy center, for NGC 4709 in 2 rings between 32 and 54". See Fig. 5 as an example. Unlike for the fainter rest of the investigated galaxies, the error of $P_{0}$ was only of the order of a few percent (see as well Table 4). This is because the area used to measure SBF was several hundred times larger.

The second difference was that the specific frequency $S_{N}$ could be calculated more precisely than the rough estimate adopted for the smaller, mainly dwarf galaxies. To do so, we obtained aperture photometry of all sources in the rings where SBF were measured. To select GCs, we demanded the sources to be unresolved, fainter than $I=21 \mathrm{mag}$ and to be in the colour range $0.8<(V-I)<1.3$ mag (Kissler-Patig et al. 1997). By doing the same photometry and applying the same selection criteria to a background field we calculated the number 

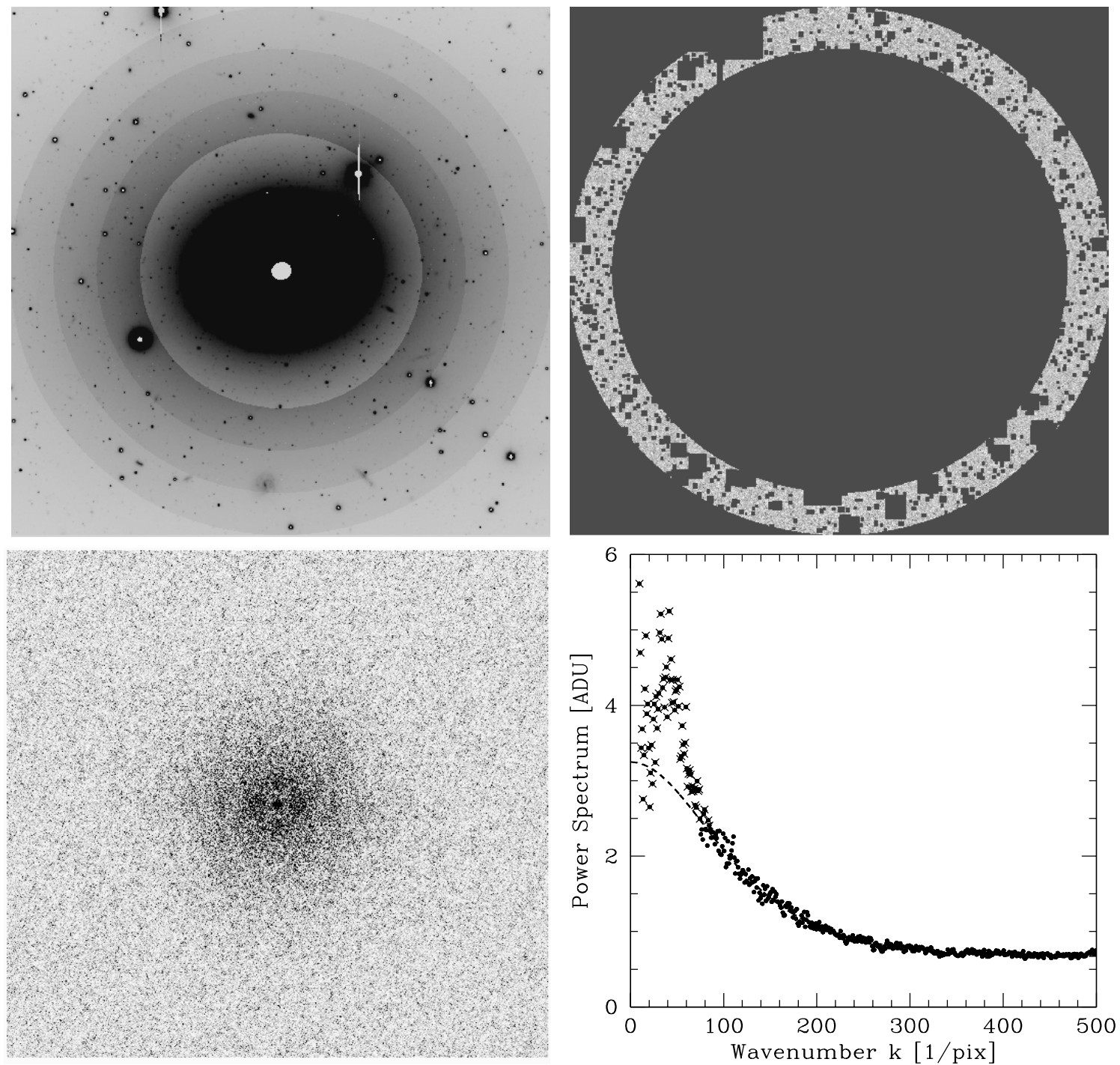

Fig. 5. Example images and plots illustrating the SBF measurement procedure for NGC 4696, the central galaxy of the Centaurus cluster. From left to right and top to bottom: 1. Original galaxy image. The three rings within which SBF were measured are indicated by different offset intensities applied to the respective rings. 2. Image containing only the outermost ring, with any contaminating objects masked. 3. twodimensional power spectrum of image 2. 4. Azimuthally average of the former image with dashed line showing the result of the fit and crosses marking the points rejected for the measurement.

density of contaminating background objects, which proved to be negligible. The simultaneous detection incompleteness for GCs in $V$ and $I$ reached $50 \%$ at typically $I=24.5 \mathrm{mag}$, a limit about 0.5 mag brighter than for detection only in the $I$-band. A Gaussian was fit to the incompleteness corrected $G C$ luminosity function until the $50 \%$ completeness limit. The error of the fitted parameters turn-over magnitude (TOM) and total number of $G C$ s was calculated by varying the width $\sigma$ by \pm 0.2 mag around a mean of $1.3 \mathrm{mag}$. This was done due to the well known fact that TOM and $\sigma$ are covariant. 1.3 mag is the mean $\sigma$ from Kundu \& Whitmore's (2001) HST results on the GCSs of a large sample of early-type galaxies, and $0.2 \mathrm{mag}$ is the standard deviation of their results.

The results of our GCLF measurements are given in Table 3. For both galaxies, the TOMs are brighter than the incompleteness limit. For NGC 4696, the derived $S_{N}$ is $4.4 \pm$ 0.8 , for NGC 4709 it is $2.1 \pm 0.5$. This results in $\Delta G C=0.08$ for NGC 4696 and $\Delta G C=0.02$ for NGC 4709 (see Table 4). Note that the values for $S_{N}$ refer only to the ring-regions where SBF were measured, not to the entire galaxies. For NGC 4696, our value is consistent with the result of Lee \& Geisler (1997 and Lee, private communication), who obtain $S_{N}=6 \pm 1$. For NGC 4709, our result is consistent with the mean $S_{N}=2.4 \pm$ 1.8 obtained by Kundu \& Whitmore (2001) from HST investigations of 28 nearby early type giants.

In a forthcoming paper (Hilker \& Mieske, in prep.), the $G C$ systems of NGC 4696 and NGC 4709 will be dealt with in more detail.

\section{Results}

The results of all SBF measurements are summarized in Table 4 and plotted in Figs. 6 to 8. To calculate the k-correction for the galaxies with no measured radial velocity available in the 
Table 3. Details of the $S_{N}$ measurement for the two giant Centaurus members NGC 4696 and NGC 4709. Note that this measurement is restricted only to the rings where SBF were measured. $(m-M)_{G C}$ is calculated assuming -8.5 mag as the absolute $I$ band TOM (Kundu \& Whitmore 2001). $I_{\text {cut }}$ is the limiting magnitude for the GCLF fitting. $N_{G C \text {,rings }}$ is the incompleteness corrected total number of GCs in the rings where SBF were measured. $M_{V, \text { rings }}$ is the absolute magnitude in the same region. For its calculation the distance modulus adopted was the mean of the SBF and GCLF distance modulus.

\begin{tabular}{r|lllllll}
\hline \hline Gal-Nr. & TOM [mag] & $(m-M)_{G C}$ & $(m-M)_{\mathrm{SBF}}^{*}$ & $I_{\text {cut }}[\mathrm{mag}]$ & $N_{G C \text {,rings }}$ & $M_{V, \text { rings }}$ & $S_{N, \text { rings }}$ \\
\hline NGC 4696 & $24.25 \pm 0.2$ & $32.75 \pm 0.2$ & $32.84 \pm 0.14$ & 24.5 & $1140 \pm 110$ & $-21.05 \pm 0.2$ & $4.4 \pm 0.8$ \\
NGC 4709 & $23.6 \pm 0.2$ & $32.1 \pm 0.2$ & $32.36 \pm 0.15$ & 24.6 & $143 \pm 20$ & $-19.60 \pm 0.2$ & $2.1 \pm 0.5$ \\
\hline
\end{tabular}

${ }^{*}$ From Table 4.

Table 4. Result of the SBF measurements for the investigated Centaurus cluster galaxies. $Z P, \Delta G C, \bar{m}_{I}$ and $(m-M)$ are given in magnitudes. The error in $(m-M)$ is the quadratic sum of the error in $\bar{m}_{I}$ and in deriving $\bar{M}_{I}$ from $(V-I)$ (see text). The distance error is the mean of the upper and lower distance error range corresponding to the magnitude error in $(m-M)$. In the lowest row, the mean distance $d$ is given.

\begin{tabular}{|c|c|c|c|c|c|c|c|c|c|}
\hline CCC-Nr. & Field & $\bar{P} P_{0}[\mathrm{ADU}]$ & 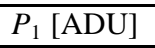 & $\overline{Z P}$ & $\overline{S / N}$ & 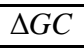 & $\overline{\bar{m}_{\mathrm{I}}}$ & $\overline{(m-M)}$ & $\overline{d[\mathrm{Mpc}]}$ \\
\hline 52 & 1 & $2.81 \pm 1.18$ & 0.64 & 32.76 & 4.39 & 0.15 & $31.35 \pm 0.43$ & $33.34 \pm 0.45$ & $46.7 \pm 9.7$ \\
\hline 61 & 1 & $2.94 \pm 0.76$ & 0.51 & 32.76 & 5.77 & 0.09 & $31.24 \pm 0.27$ & $33.01 \pm 0.30$ & $40.0 \pm 5.5$ \\
\hline 65 (N4696) & 1 & $2.33 \pm 0.04$ & 0.49 & 32.78 & 4.78 & 0.08 & $31.50 \pm 0.07$ & $32.84 \pm 0.14$ & $37.0 \pm 2.4$ \\
\hline 70 & 1 & $3.56 \pm 0.93$ & 0.32 & 32.76 & 11.12 & 0.02 & $30.98 \pm 0.27$ & $32.30 \pm 0.29$ & $28.8 \pm 3.9$ \\
\hline 75 & 1 & $3.30 \pm 1.39$ & 0.86 & 32.77 & 3.84 & 0.19 & $31.48 \pm 0.44$ & $33.56 \pm 0.45$ & $51.5 \pm 10.9$ \\
\hline 89 & 2 & $2.16 \pm 0.56$ & 0.29 & 32.78 & 7.45 & 0.28 & $31.78 \pm 0.32$ & $33.51 \pm 0.34$ & $50.3 \pm 8.0$ \\
\hline 111 & 3 & $6.02 \pm 1.56$ & 0.46 & 32.67 & 13.09 & 0.03 & $30.31 \pm 0.27$ & $32.67 \pm 0.29$ & $34.2 \pm 4.6$ \\
\hline 115 & 3 & $4.02 \pm 1.69$ & 1.34 & 32.67 & 3.00 & 0.09 & $30.81 \pm 0.43$ & $33.24 \pm 0.44$ & $44.5 \pm 9.2$ \\
\hline 121 & 3 & $2.65 \pm 1.11$ & 0.76 & 32.67 & 3.49 & 0.15 & $31.28 \pm 0.43$ & $33.37 \pm 0.45$ & $47.2 \pm 9.8$ \\
\hline 123 & 3 & $5.38 \pm 2.26$ & 0.62 & 32.67 & 8.68 & 0.03 & $30.39 \pm 0.42$ & $32.66 \pm 0.44$ & $34.1 \pm 7.0$ \\
\hline 124 & 3 & $6.28 \pm 2.64$ & 3.36 & 32.67 & 1.87 & 0.02 & $30.26 \pm 0.42$ & $33.02 \pm 0.56$ & $40.2 \pm 10.6$ \\
\hline 130 (N4709) & 3 & $1.90 \pm 0.10$ & 0.38 & 32.67 & 5.00 & 0.02 & $31.51 \pm 0.08$ & $32.36 \pm 0.15$ & $29.6 \pm 2.0$ \\
\hline 125 & 4 & $5.28 \pm 1.37$ & 0.47 & 32.78 & 11.23 & 0.24 & $30.78 \pm 0.30$ & $32.82 \pm 0.33$ & $36.6 \pm 5.5$ \\
\hline 58 & 5 & $2.62 \pm 1.1$ & 0.92 & 32.72 & 2.85 & 0.21 & $31.44 \pm 0.44$ & $33.75 \pm 0.46$ & $56.2 \pm 11.9$ \\
\hline 68 & 6 & $5.03 \pm 2.11$ & 4.13 & 32.72 & 1.22 & 0.06 & $30.59 \pm 0.42$ & $33.15 \pm 0.47$ & $32.7 \pm 9.2$ \\
\hline
\end{tabular}

$41.3 \pm 2.1$

literature, $3000 \mathrm{~km} \mathrm{~s}^{-1}$ was assumed. The error of $(m-M)$ in the table is the quadratic sum of the error in measuring $\bar{m}_{I}$ and in deriving $\bar{M}_{I}$ from $(V-I)$ (see Sect. 3.2.3). The error in metric distance $d$ is the mean of the upper and lower distance error range corresponding to the magnitude error in $(m-M)$.

\subsection{Distance to the Centaurus cluster and its subcomponents}

The mean distance of all investigated galaxies is $41.3 \pm$ $2.1 \mathrm{Mpc}$, corresponding to $33.08 \pm 0.11 \mathrm{mag}$ in distance modulus. Our result is higher than Tonry's result derived from their SBF survey, which was $32.63 \pm 0.09$ mag based on SBF measurements of 5 Cen 30 and 3 Cen 45 early-type giants (SBF IV). Although Dressler (1993) was the first to publish SBF-distances to Centaurus cluster galaxies, we will in the following compare our results only with Tonry's newer values, as they have refined and complemented Dressler's early measurements.

For the 2 galaxies common to both our and Tonry's data set, namely NGC 4696 and NGC 4709, Tonry et al. derive $(m-M)=32.75 \pm 0.17 \mathrm{mag}$ for NGC 4696 and $(m-M)=$ $32.74 \pm 0.23$ for NGC 4709 . To find out whether our results are consistent with that, we quadratically subtract the cosmic scatter error contribution of $0.1 \mathrm{mag}$ from our distance error, as we compare the same galaxies. We then get $32.84 \pm 0.10 \mathrm{mag}$ for NGC 4696 and $32.36 \pm 0.11$ mag for NGC 4709. Tonry's and our distance for NGC 4696 agree very well, while the distances for NGC 4709 differ by 1.1 sigma, or almost 0.4 mag. A further discussion of the mean difference in distance between our and Tonry's Cen 30 sample is given in Sect. 5 .

\subsubsection{Cen 30 and Cen 45 distance}

We separate our sample galaxies according to their radial velocity into Cen $30\left(v_{\mathrm{rad}}<4580 \mathrm{~km} \mathrm{~s}^{-1}\right)$ and Cen $45\left(v_{\mathrm{rad}}>\right.$ $4580 \mathrm{~km} \mathrm{~s}^{-1}$ ), following Stein et al. (1997). We get 8 galaxies in Cen 30 and 3 in Cen 45.4 of our 15 galaxies do not have a radial velocity measured. In Fig. 7 it can be verified that there is no obvious distance separation between the two subsamples. The distance modulus corresponding to the mean distance of our 8 Cen 30 galaxies is $33.11 \pm 0.17 \mathrm{mag}$, of our 3 Cen 45 galaxies it is $32.84 \pm 0.29 \mathrm{mag}$. The distance moduli difference $(m-M)_{\text {Cen } 45}-(m-M)_{\text {Cen } 30}$ is $-0.27 \pm$ 0.34 mag, consistent with both subclusters being at the same distance, but allowing for a considerable range of separations between $0.07 \mathrm{mag}$ and $-0.61 \mathrm{mag}$. This rules out that the subclusters are separated by their Hubble flow distance of 


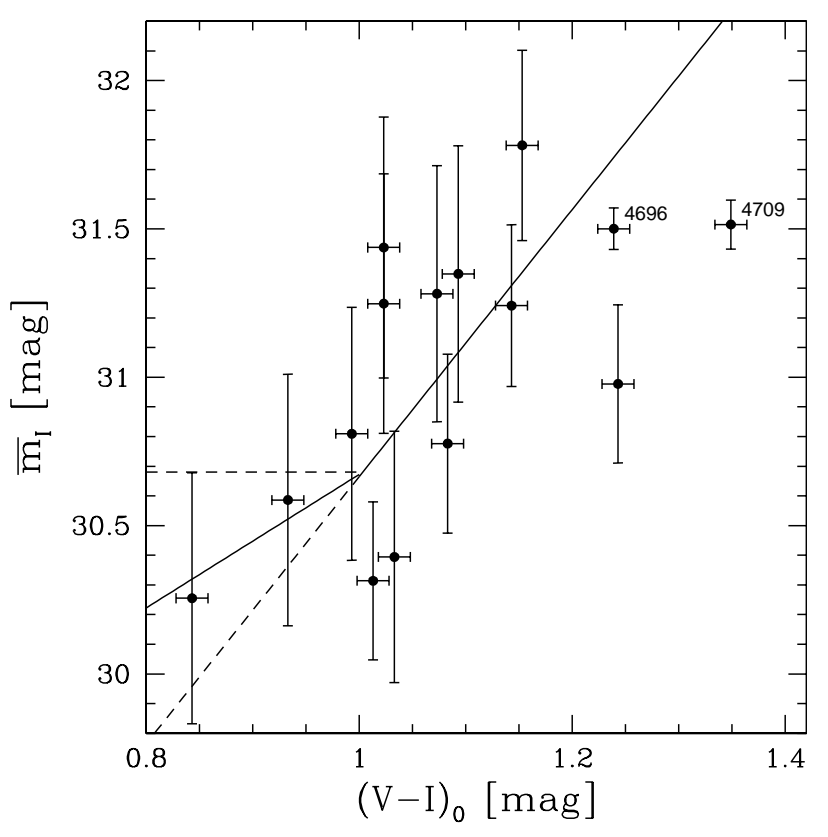

Fig. 6. Colour-SBF diagram of the investigated Centaurus cluster galaxies. The location of NGC 4696 and NGC 4709 is indicated by their NGC number. The solid line corresponds to Eqs. (1) $((V-I)>$ $1.0)$ and (2) $((V-I) \leq 1.0)$ for a distance modulus of $(m-M)=$ $33.08 \mathrm{mag}$, the mean distance of the investigated galaxies. Dashed lines as in Fig. 1. The errors in $(V-I)$ are estimated from the uncertainty in the local sky level determination for $V$ and $I$. For NGC 4696 and NGC 4709 , the errors in $\bar{m}_{I}$ are estimated from the scatter in $\bar{m}_{I}$ between the different investigated rings (Sect. 3.2.4). For the other galaxies they are estimated from Monte Carlo simulations (Sect. 3.2.3).

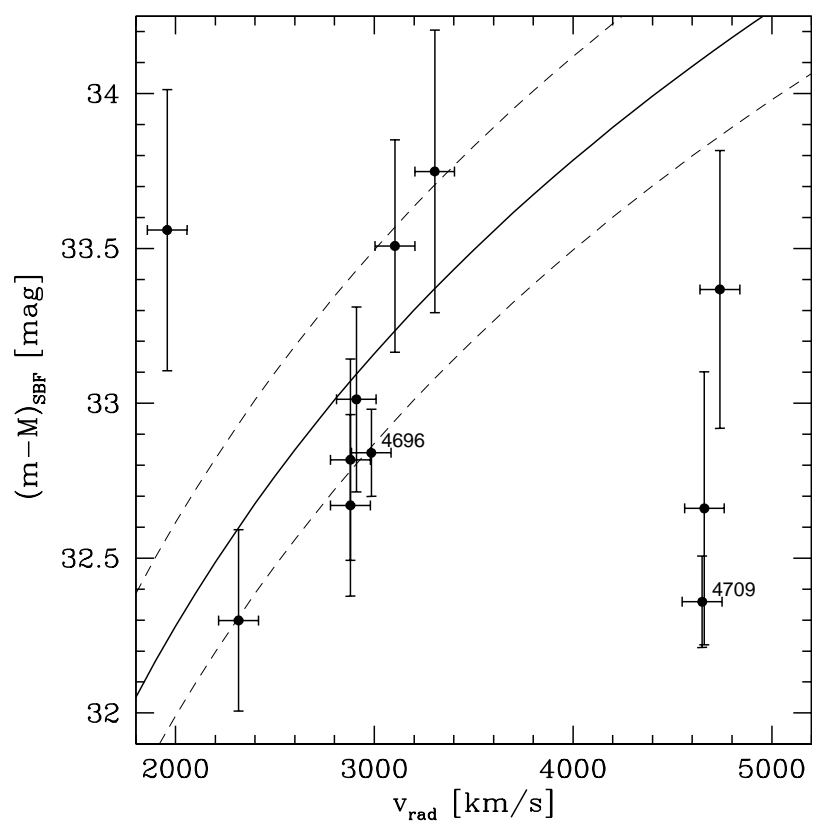

Fig. 7. Heliocentric radial velocity is plotted vs. distance modulus for the investigated galaxies with measured redshifts. The location of NGC 4696 and NGC 4709 is indicated by their NGC number. The solid line gives the Hubble flow for $H_{0}=70 \mathrm{~km} \mathrm{~s}^{-1} \mathrm{Mpc}^{-1}$. The upper dashed line corresponds to $H_{0}=60$, the lower dashed line to $H_{0}=80$. The fact that the data points are not aligned along these lines but rather show no correlation indicates that the galaxies are either bound within one cluster or are situated in a region with gravitational distortion.

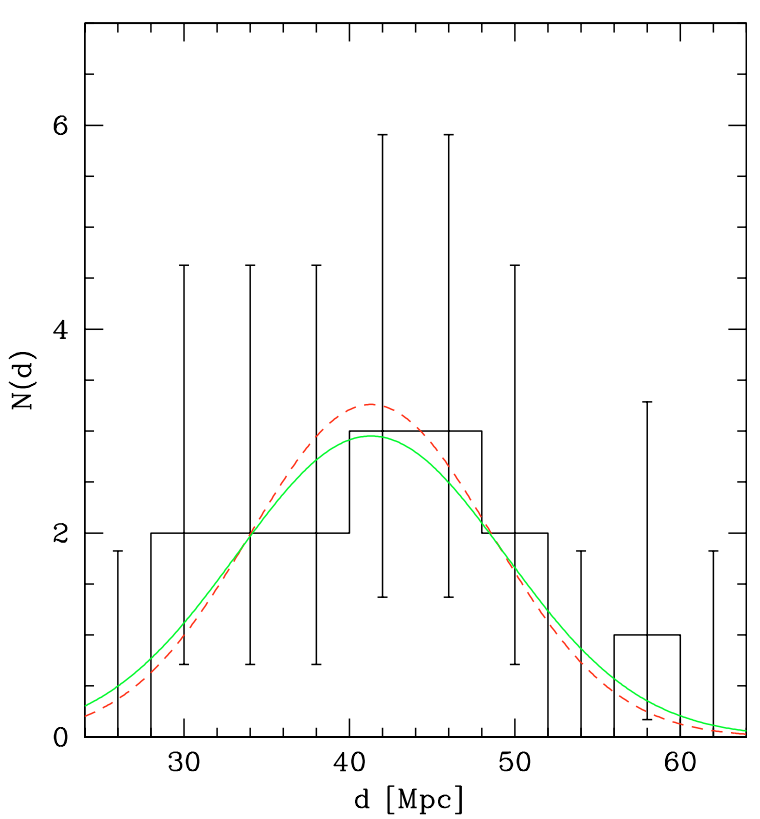

Fig. 8. Histogram of the SBF distances for the investigated Centaurus Cluster galaxies. Note that the error bars do not correspond to the square root of the bin value, but are calculated based on the formulae given by Gehrels (1986) for the calculation of errors for low number statistics. The solid line represents a gaussian distance histogram centered on the mean $41.3 \mathrm{Mpc}$ with a half-width of $8.1 \mathrm{Mpc}$, corresponding to the standard deviation of the measured distances around their mean. The dashed line represents a Gaussian distance histogram with half-width $7.3 \mathrm{Mpc}$, which is the mean single distance measurement uncertainty (see text and Table 4).

about +0.9 mag, and even favours Cen 45 being closer than Cen 30. Comparing our results with Tonry's $32.53 \pm 0.11 \mathrm{mag}$ for Cen 30 and $32.81 \pm 0.09$ mag for Cen 45 shows that only for Cen 45 are the results consistent within the error ranges. For Cen 30, Tonry get a 0.58 mag shorter distance at a $2.1 \sigma$ significance. We argue that this discrepancy is due to selection effects within the Tonry sample, as will be pointed out in more detail in Sect. 5.

It is worth taking a special look at the distances to the two central galaxies of Cen 30 (NGC 4696) and Cen 45 (NGC 4709). Our measurements place NGC $46960.48 \pm$ 0.21 mag more distant than NGC 4709, at a $2.3 \sigma$ significance the distances are different. The NGC 4696 distance is consistent with the mean distance of the whole sample and the mean distance of the 8 Cen 30 galaxies. The NGC 4709 distance is only consistent with the Cen 45 distance, but is shorter than the Cen 30 distance and the distance of the whole sample. This might indicate that the distance difference found between the Cen 30 and Cen 45 sample is a real one. Due to the large uncertainties involved in the distance measurement for the rest of our sample galaxies, the distance difference between the Cen 30 and Cen 45 central galaxies NGC 4696 and NGC 4709 is a more precise indicator of the Cen 30-Cen 45 distance than the mean difference between the entire Cen 30 and Cen 45 sample, assuming that NGC 4696 and NGC 4709 are located at the respective center of the two components. The significant distance difference is supported by the investigations of their 
globular cluster systems (Sect. 3.2.4), which show a difference of $0.65 \pm 0.28$ mag between the two TOMs, placing NGC 4696 further than NGC 4709.

One can then interpret this separation within the clustersubcluster scenario such that Cen 45 is a subgroup falling into Cen 30 but not having reached it yet. In order not to base such a conclusion only on measurements of the two main galaxies, SBF measurements from more giant Cen 30 and Cen 45 members are needed.

\section{2. $H_{0}$}

The well known peculiar velocity of the Local Group towards the GA of $300 \pm 100 \mathrm{~km} \mathrm{~s}^{-1}$ (SBF II) allows a derivation of the Hubble constant $H_{0}$ from our distance measurements. Due to the large velocity dispersion of almost $1000 \mathrm{~km} \mathrm{~s}^{-1}$ observed for Cen 30 galaxies (Stein et al. 1997), we prefer to adopt the mean heliocentric radial velocity $3170 \pm 174 \mathrm{~km} \mathrm{~s}^{-1}$ of the 74 early type Cen 30 galaxies investigated by Stein et al. rather than the mean $2790 \mathrm{~km} \mathrm{~s}^{-1}$ of the 8 Cen 30 members investigated by us, as the former velocity is much better defined because of its large underlying sample size. The difference in mean radial velocity between our sample and the Stein et al. sample is about $380 \mathrm{~km} \mathrm{~s}^{-1}$. This difference lies well within the range of statistical fluctuations, since with a sample of 8 galaxies and a velocity dispersion of $1000 \mathrm{~km} \mathrm{~s}^{-1}$, the accuracy of the mean is of the order of $350 \mathrm{~km} \mathrm{~s}^{-1}$. If we correct for the peculiar motion towards the GA, which we adopt to be at the location of the Centaurus cluster, we get $3470 \pm 200 \mathrm{~km} \mathrm{~s}^{-1}$ as the mean Hubble flow velocity. The mean distance of the 8 Cen 30 members is $41.8 \pm 3.4 \mathrm{Mpc}$. The resulting value for the Hubble constant is then $H_{0}=83.0 \pm 8.3 \mathrm{~km} \mathrm{~s}^{-1} \mathrm{Mpc}^{-1}$.

The most precise current value for $H_{0}$ comes from the WMAP (Wilkinson Microwave Anisotropy Probe)-team (Bennett et al. 2003; Spergel et al. 2003), who give $H_{0}=$ $71_{-3}^{+4} \mathrm{~km} \mathrm{~s}^{-1} \mathrm{Mpc}^{-1}$. Our value of $83.0 \pm 8.3 \mathrm{~km} \mathrm{~s}^{-1} \mathrm{Mpc}^{-1}$ agrees marginally with theirs.

Assuming the WMAP value $H_{0}=71 \mathrm{~km} \mathrm{~s}^{-1} \mathrm{Mpc}^{-1}$ and the Cen 30 distance of $41.8 \pm 3.4 \mathrm{Mpc}$ derived by us, the undisturbed Hubble flow velocity at that distance would be $2970 \pm$ $280 \mathrm{~km} \mathrm{~s}^{-1}$. This is remarkably consistent both with the mean heliocentric velocity of our 8 galaxies as well as of the much larger sample of Stein et al. (1997). It indicates that the peculiar velocities of the Centaurus cluster galaxies with respect to the Hubble flow might be much smaller than previously found by Tonry et al. (SBF II), which would result in smaller infall velocities into and hence a smaller mass for the Great Attractor. This will be discussed in more detail in Sect. 5.5.

\section{Discussion}

\subsection{Comparison with Tonry, Tonry's selection effects}

The distances to the two galaxies in common to both Tonry's and our data set agree to within their errors for NGC 4696 and differ with $1.1 \sigma$ significance for NGC 4709. Our results place NGC $47090.48 \pm 0.21$ mag closer than NGC 4696, while Tonry et al. obtain practically the same distance for both galaxies. The significant separation derived from our data is supported by the investigation of their GCSs.

We believe that the difference between our and Tonry's distance for NGC 4709 is caused by the different cutoff magnitudes for investigating the globular cluster systems. While we are able to map both GCSs well beyond their TOM (down to 25 mag in $I$ ), Tonry et al. have a significantly brighter dereddened cutoff-magnitude for their investigations, which is $23.6 \pm$ $0.2 \mathrm{mag}$ for NGC 4696 and $23.8 \pm 0.3 \mathrm{mag}$ for NGC 4709 in $I$ (Blakeslee \& Tonry, private communication). They obtain a contribution close to $50 \%$ to the SBF-signal from undetected globular clusters, which causes an additional distance error of almost the same order (Blakeslee \& Tonry, private communication). The fact that NGC 4709 has a very poor GCS according to our deep data, could have made Tonry et al. overestimate the $G C$ contributions for NGC 4709, as they only map it down to the TOM. In that case, they would have subtracted too much $G C$ contribution from their SBF signal, yielding a too weak SBF amplitude and consequently a too large distance.

An overall bias in the sense that Tonry's distances for their faintest survey galaxies might be too close has been discussed by SBF IV and Blakeslee et al. (2002), already. They argue that a combination of two factors makes them obtain too small distances at their faint survey limit, which was at about the Centaurus cluster redshift.

First, for a selection effect such that at a given cluster in which the SBF signal of the member galaxies is just at the limit of being detectable, one will only measure those whose observational errors place them above the detection limit and one will not measure those below the limit. Second, a "Malmquist bias" (Malmquist 1920; Lynden-Bell 1988), which referes to the distance bias arising from the spatial distribution of the sample galaxies, including the increase in the volume element with distance. This bias is proportional to the measured distance error and therefore stronger at the faint survey limits. Blakeslee et al. (2002) find that both of these effects are interrelated. They correct their measured distances for these selection effects and obtain an overall correction of about $0.3 \mathrm{mag}$ towards larger distances for the Centaurus cluster (Blakeslee $\&$ Tonry, private communication). Thus, their mean Centaurus distance becomes about $32.9 \mathrm{mag}$, which agrees well with our result. Note that the galaxy distances based upon which the mass of the Great Attractor and the corresponding Hubble flow distortion was calculated in $S B F I I$ were not corrected for this bias, indicating a possible overestimation of peculiar velocities into the GA and hence its mass. See Sect. 5.5 for further discussion.

\subsection{Systematic effects in our data?}

Are there significant systematic selection effects present in our data?

In our final data set, we include three galaxies with $\mathrm{S} / \mathrm{N}$ smaller than 3, whose mean distance is $46.4 \pm 5 \mathrm{Mpc}$. Excluding them from the sample lowers the average distance by $1.7 \mathrm{Mpc}$, which is below significance. This does not indicate the presence of a strong selection effect as mentioned in 
the former section. An additional test is whether we can see a correlation between $(m-M)$ and $M_{V}$ for the investigated galaxies. Assuming that we probe galaxies at the same distance, then the above mentioned selection effect would move the faintest galaxies observed to closer distances. Figure 9 shows such a plot. There is no dependence between $(m-M)$ and $M_{V}$ within our error ranges. If a systematic effect is present, it is negligible compared to the measurement uncertainty.

The same plot serves to check for an overall distance difference between giant and dwarf galaxies. Separating the investigated galaxies by magnitude into giants $\left(M_{V} \leq-17 \mathrm{mag}\right)$ and dwarfs $\left(M_{V} \geq-17 \mathrm{mag}\right.$ ) (Hilker et al. 2003), we get a mean distance of $39 \pm 6 \mathrm{Mpc}$ for the giants and 41.9 $\pm 2.3 \mathrm{Mpc}$ for the dwarfs, i.e. there is no significant distance difference between giants and dwarfs.

\subsection{Distance to NGC 4709, Cen 30-Cen 45 separation}

In Fig. 10, we plot $(m-M)$ vs. $(V-I)$. Here, a correlation would hint at systematic errors in derivation of $\bar{M}_{I}$ from $(V-I)$, under the assumption that all investigated galaxies are at the same distance. Indeed, there seems to be a trend towards smaller distances for redder galaxies. This trend is, however, almost entirely defined by the reddest investigated galaxy NGC 4709 . Including the NGC 4709 data point, there is a linear relation between $(m-M)$ and $(V-I)$ with slope different from zero with $3.4 \sigma$ significance. Excluding it, the significance drops to $1.45 \sigma$. For comparison, excluding the bluest data point only slightly increases the significance to $3.7 \sigma$.

The issue condenses to the question: Is the small distance for NGC 4709, placing it $7.5 \pm 3 \mathrm{Mpc}$ closer to us than NGC 4696, due to an unusual stellar population causing a bias in deriving $\bar{M}_{I}$ from $(V-I)$, or, is the small distance a real physical fact? A real small distance is supported by the investigation of NGC 4709's and NGC 4696 's GCS. It reproduces both the absolute distance of both galaxies and their distance difference derived by SBF, see Table 3. Theoretical stellar population models (see for example Fig. 1 or Mieskel), although they frequently predict offset $\bar{M}_{I}$ values compared to Eq. (1), do not allow for a strong scatter in $\bar{M}_{I}$ at a given $(V-I)$ for red colours, which as well favours a real small distance instead of a stellar population effect mimicking it.

We therefore believe that the scenario of NGC 4709 being at a closer distance than NGC 4696 is more probable than NGC 4709's bright SBF magnitude being caused by unusual stellar populations. Note that the distance difference between NGC 4709 and NGC 4696 in combination with their velocity difference is consistent with the Hubble flow distortion caused by the Great Attractor, if one assumes that NGC 4696 with Cen 30 is located at the GA's center. In the lower panels of Figs. 18 and 19 of SBF II the distortion of the Hubble flow along the line of sight towards the GA is shown as a function of distance. There are two distance ranges with respect to the GA's center (at $43 \mathrm{Mpc}$ ) where a radial velocity of $4500 \mathrm{~km} \mathrm{~s}^{-1}$ is reached: one between approximately -10 and $-2 \mathrm{Mpc}$ in front of the GA and one at about $+20 \mathrm{Mpc}$ behind. The former range

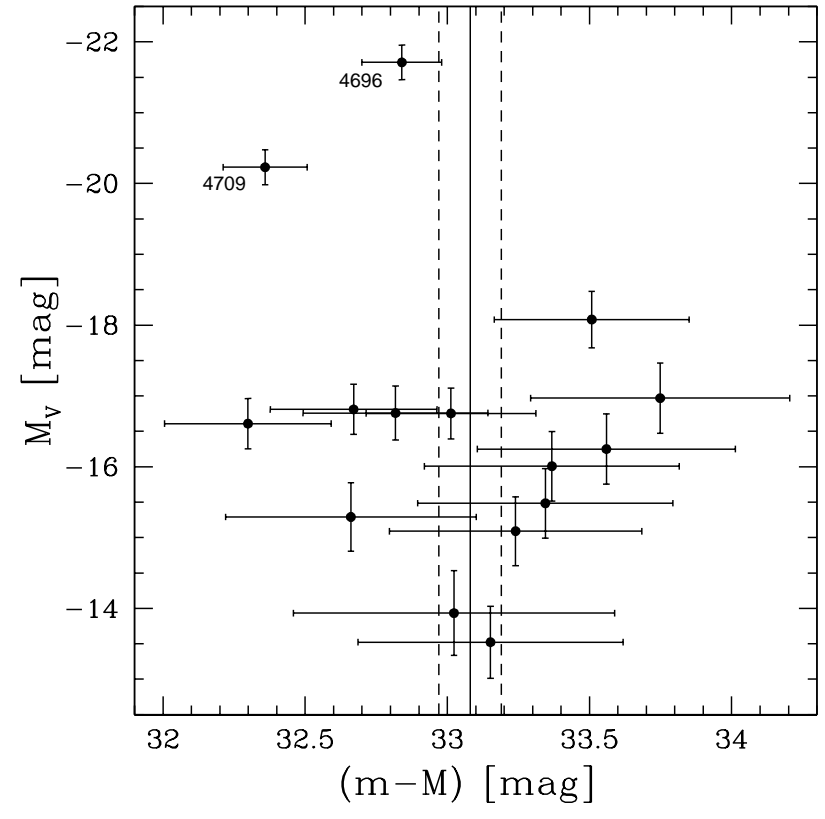

Fig. 9. Distance modulus $(m-M)$ of the investigated galaxies plotted vs. their absolute magnitude $M_{V}$. The solid vertical line indicates the mean distance of all galaxies. The dashed lines indicate the error range of the mean distance. 4 of the 15 investigated galaxies fall outside of this error range.

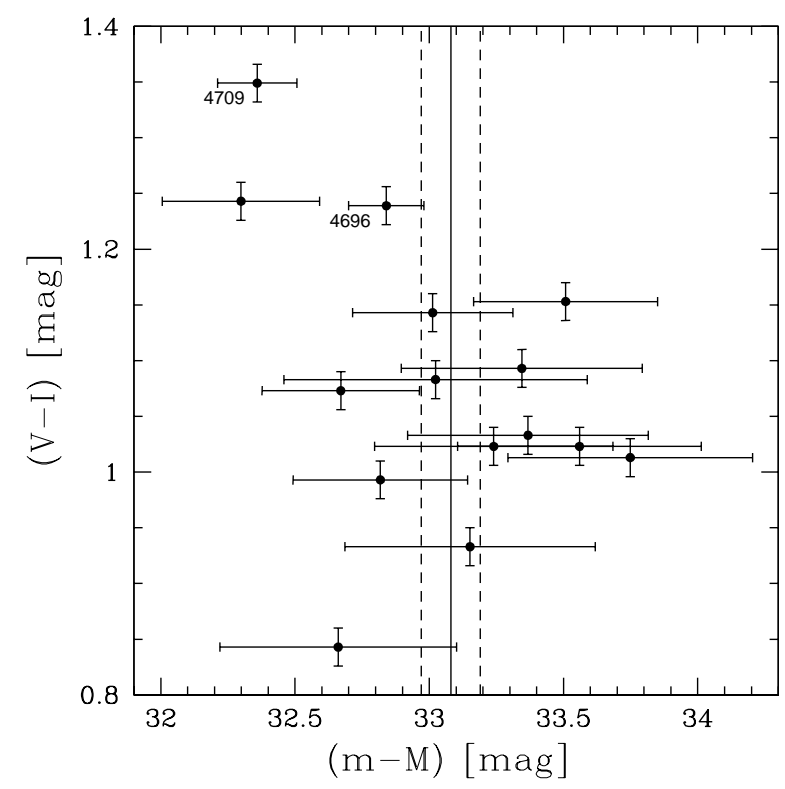

Fig. 10. Distance modulus $(m-M)$ of the investigated galaxies plotted vs. their colour $(V-I)$. Vertical lines as in Fig. 9.

corresponds to the approximately sinusoidally shaped Hubble flow distortion pattern in front of the GA. The latter value corresponds to the undisturbed Hubble flow.

With the radial velocity difference of about $1500 \mathrm{~km} \mathrm{~s}^{-1}$ and the distance difference of about $7.5 \pm 3 \mathrm{Mpc}$ between NGC 4696 and NGC 4709 one can calculate the time difference left until NGC 4709 reaches the same distance as NGC 4696, assuming that the infall velocity does not change considerably over time. The result is $5 \pm 2$ Gyrs. This is a very large value, 
which is not consistent with an ongoing merger scenario between Cen 30 and Cen 45 in which NGC 4709 participates, as for example proposed by Churazov et al. (1999) based on an X-ray temperature map of the central Centaurus cluster. However, Furusho et al. (2001) have suggested based on improved X-ray data that a major merger is not taking place right now but already occured several Gyrs ago. Our finding that NGC 4709 is falling into Cen 30 but has not yet reached it yet then suggests that NGC 4709 has not been involved with a merger event during the last few Gyrs, but might be the subject of the next merger to come in several Gyrs more. Nevertheless, we cannot exclude that NGC 4709 has passed by NGC 4696 in the more distant past.

\subsubsection{Filamentary structure}

It has been suggested by Churazov et al. (1999) that in the direction of the Centaurus cluster we are looking into a large scale filamentary structure. They bring this scenario forward in order to explain an unusually extreme " $\beta$-problem", i.e. a substantial disagreement between the energy-ratio per unit mass for galaxies to that in the gas derived from X-ray temperatures and from galaxy velocity dispersion. Colberg et al. (1999) have shown in numerical simulations that clusters accrete matter from a few preferred directions, defined by filamentary structures, and that the accretion persists over cosmologically long times.

This scenario of a filamentary structure is supported by the radial distance difference of $7.5 \pm 3 \mathrm{Mpc}$ between NGC 4696 and NGC 4709 derived by us, compared to their small angular separation of about 0.25 degrees or $0.2 \mathrm{Mpc}$ projected distance.

Precise SBF-distances to more Centaurus cluster giants must be measured to prove a filamentary structure along the line of sight towards Centaurus. Related to this subject, in the next section an upper limit on the depth of the investigated Centaurus cluster portion is derived, discussing as well the distance scatter observed by Tonry et al. (SBF IV).

\subsection{Depth of the Centaurus cluster}

As can be seen in Fig. 8, the measured distance scatter of our data is almost equal to the mean single measurement uncertainty, allowing no derivation of a lower limit for the Centaurus cluster's depth. We can derive an upper limit for the depth, though. We apply the inequality $\frac{(n-1)^{2}(\Delta x)^{2}}{\chi_{1-\frac{\alpha}{2}}^{2}} \leq \sigma^{2} \leq \frac{(n-1)^{2}(\Delta x)^{2}}{\chi_{\frac{\alpha}{2}}^{2}}$ to obtain the confidence interval for the real variance $\sigma^{2}$ of a distribution with a measured variance $\Delta x^{2}$. In this inequality, the error probability of the confidence interval is given by $1-\alpha . n-1$ denotes the number of degrees of freedom, in our case $n-1=14$. From tabulated $\chi^{2}$ values we find that the Centaurus cluster would have to be radially extended over more than $10 \mathrm{Mpc}$ to both sides in order to exclude with more than $95 \%$ confidence a $\delta$-distribution for the distance of our sample galaxies.

We therefore derive a formal upper limit of $\pm 10 \mathrm{Mpc}$ radial extension for the Centaurus cluster. The cluster's angular extension on the sky of 6 degrees (Lucey et al. 1986) corresponds to a diameter of 4.3 Mpc at 41.3 Mpc distance. This would make us expect a distance scatter of about $2 \mathrm{Mpc}$ around the mean in case of a spherical cluster shape. I.e. we are only sensitive to a cigar-shape with the major axis at least five times larger than the minor axis, leaving enough space for a possible filamentary structure as proposed in Sect. 5.3.1.

Tonry et al. (SBF IV) measure SBF-distances for a total of 8 Centaurus cluster giants. Their mean distance is $33.7 \mathrm{Mpc}$ with a rms-scatter of $3.9 \mathrm{Mpc}$ and a mean measurement error of $5.3 \pm 1.2 \mathrm{Mpc}$. The distance scatter is even smaller than the measurement error at about $1 \sigma$ significance. I.e. with a measurement accuracy about $30 \%$ better than ours, Tonry et al. do not find a significant radial extension of the Centaurus cluster. However, Tonry et al. only have a sample of 8 galaxies instead of the 15 galaxies in our sample, which weakens the statistical significance by about the same factor of $30 \%$.

Therefore, the upper limit for the cluster's depth derived by us cannot be improved with their data. Besides, the selection effect inherent in Tonry's data will generally decrease the distance scatter, as those objects for which the observational errors give a larger distance are more likely to be excluded from their survey.

\subsection{Overestimation of the Great Attractor mass?}

The very large GA mass of almost $10^{16} M_{\text {sun }}$ derived in SBF II was partially a consequence of the large peculiar velocities observed by Tonry et al. for the Centaurus cluster galaxies, which implied a very strong gravitational pull into the GA. Using their mean Cen 30 distance of $32.0 \pm 1.8 \mathrm{Mpc}$ and $H_{0}=71 \mathrm{~km} \mathrm{~s}^{-1} \mathrm{Mpc}^{-1}$ as derived by the WMAP team, the Hubble flow velocity for Cen 30 would be $2270 \pm 180 \mathrm{~km} \mathrm{~s}^{-1}$, $900 \mathrm{~km} \mathrm{~s}^{-1}$ smaller than the mean heliocentric radial velocity $3170 \pm 170 \mathrm{~km} \mathrm{~s}^{-1}$ of the Stein et al. (1997) sample of early type Centaurus cluster galaxies. Taking into account the $300 \mathrm{~km} \mathrm{~s}^{-1}$ peculiar velocity of the Local Group towards Centaurus (SBF II), the peculiar velocity of Cen 30 even becomes $1200 \pm 270 \mathrm{~km} \mathrm{~s}^{-1}$. However, already Blakeslee et al. (2002) noted that the distances at the faint limit of Tonry et al.'s survey are systematically underestimated by about 0.3 mag suggesting smaller peculiar velocities for Centaurus, a bias which had not been taken into account for the derivation of the GA mass in SBF II.

With our new distance value of $41.8 \pm 3.4 \mathrm{Mpc}$ for Cen 30, the Hubble flow velocity of Cen 30 becomes $2970 \pm$ $280 \mathrm{~km} \mathrm{~s}^{-1}$, yielding a not significant Cen 30 peculiar velocity of $200 \pm 330 \mathrm{~km} \mathrm{~s}^{-1}$. Even when including the Local Group peculiar motion, the peculiar velocity of Cen 30 is only $500 \pm$ $340 \mathrm{~km} \mathrm{~s}^{-1}$.

Our results thus imply that the Hubble flow distortion in the direction of the Centaurus cluster is smaller by a factor of 2 or more compared to the distortion obtained by Tonry et al. and might even be negligible. There are two interpretations for this: First, the mass of the GA has been significantly overestimated in SBF II. Second, the Centaurus cluster falls into the Great Attractor with a vector almost perpendicular to the line of sight. Then, the radial component of its peculiar velocity would 
be comparably small. The distance of $41.8 \pm 3.4 \mathrm{Mpc}$ to Cen 30 derived by us is very close to the GA distance of $43 \pm 3 \mathrm{Mpc}$ derived in $(S B F I I)$. In addition, Tonry and collaborators find that the Centaurus galaxies pass above the GA by about 15 degrees, which corresponds to about $10 \mathrm{Mpc}$ at the Centaurus cluster distance. Both findings are consistent with the second interpretation. Note, however, that the distance underestimation for galaxies at the distance limit of Tonry's survey would probably increase the GA distance if corrected for.

To determine whether and to what degree either a mass overestimation of the GA or a mainly tangential infall of the Centaurus cluster into the GA are responsible for the much smaller Hubble flow distortion of Centaurus cluster galaxies observed by us compared to $S B F I I$, high resolution $\mathrm{SBF}$-distances to galaxies in a much larger sky region than covered by us would be necessary.

\subsection{Backside infall?}

It has been claimed (Dressler \& Faber 1990) and disclaimed (Mathewson et al. 1992) that in the direction of the GA there is an anticorrelation between redshift and distance. Such a backand frontside infall pattern would be expected if the galaxies in front of the GA are drawn away from us, while the galaxies on the back side of the GA are drawn towards us (both falling into the GA). Can we verify such a behaviour from our data? Looking at Fig. 7 shows that there is certainly no correlation between redshift and distance, but neither there is any evidence for a significant anticorrelation. The mean radial velocity of the 4 galaxies whose distance is larger than the mean distance is $3280 \mathrm{~km} \mathrm{~s}^{-1}$. For the 7 galaxies which are closer it is $3330 \mathrm{~km} \mathrm{~s}^{-1}$. Thus, there is no correlation between distance and velocity when separating the sample according to distance. The distance modulus corresponding to the mean distance of our Cen 30 sample is $33.11 \pm 0.17 \mathrm{mag}$, for our Cen 45 sample it is $32.84 \pm 0.29 \mathrm{mag}$. When separating the sample according to radial velocity, there is a weak anticorrelation between redshift and distance, but not at a significant level. Only if one considers NGC 4696 and NGC 4709 alone, an anticorrelation at $2.3 \sigma$ significance is seen. This has already been discussed in Sect. 5.3 and been found consistent with the Hubble flow distortion. However, only two data points forming an anticorrelation, and this not even at $3 \sigma$, is not sufficient to claim such a phenomenon for the entire cluster population.

Based on our data we can therefore not find any evidence for back- or frontside infall. Note however that due to the large distance uncertainties and the low number of galaxies we could only detect very pronounced infall patterns which extend over several tens of Mpc along the line of sight. Besides, the sky position of the Great Attractor is deplaced by about 15 degrees with respect to the Centaurus cluster according to SBF II. The Centaurus galaxies might therefore not experience the GA's maximum gravitational pull.

\section{Summary and conclusions}

We have presented I-band SBF-measurements for 15 early type Centaurus cluster galaxies in the magnitude range
$19.6>V>11.5 \mathrm{mag}, 3$ giant and 12 dwarf galaxies. The measurements were made on deep photometric data obtained with VLT FORS1 in the $I$-band in 7 fields with a seeing between 0.4 and $0.6^{\prime \prime}$. The following results were obtained:

1. The mean distance of our investigated galaxies is $41.3 \pm$ $2.1 \mathrm{Mpc}$. This corresponds to a distance modulus of $33.08 \pm$ $0.11 \mathrm{mag}$ and places the Centaurus cluster at the same distance as the Great Attractor (see Tonry et al. 2000). We find that our Centaurus cluster distance is about 0.5 mag higher than Tonry's value (Tonry et al. 2001). This is explained by the fact that the sensitivity limit of Tonry's SBF-survey is reached at about the Centaurus distance and that therefore the galaxies whose observational errors place them further away are less likely to enter their survey.

2. Splitting our data according to their measured redshifts into Cen 30 and Cen 45, we obtain a distance difference $(m-M)_{\text {Cen } 45}-(m-M)_{\text {Cen } 30}=-0.27 \pm 0.34$ mag. This rules out both components being separated by their Hubble flow distance but still allows for a wide range of separation, including no separation at all. We do find a significant separation

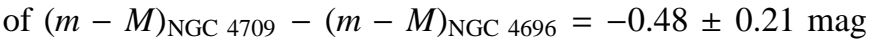
between the two dominant giants of Cen 45 and Cen 30, supported by the measured turn-over magnitudes of their respective Globular Cluster systems and supporting a scenario with Cen 45 being a subgroup falling into but not having reached Cen 30 yet. This scenario is found to be consistent with the proposed large scale filament along the line of sight towards Centaurus (Churazov et al. 1999) and the Hubble flow distortion caused by the Great Attractor (Tonry et al. 2000).

3. The Hubble constant $H_{0}$ is determined to be $83.0 \pm$ $8.3 \mathrm{~km} \mathrm{~s}^{-1} \mathrm{Mpc}^{-1}$ for our Cen 30 sample taking into account the peculiar motion of the Local Group into the direction of the Centaurus cluster.

4. The peculiar velocity of Cen 30 with respect to an undisturbed Hubble flow is $500 \pm 340 \mathrm{~km} \mathrm{~s}^{-1}$ when taking into account the peculiar motion of the Local Group into the direction of Centaurus, and only $200 \pm 330 \mathrm{~km} \mathrm{~s}^{-1}$ when not. This means a much smaller Hubble flow distortion in the direction of Centaurus than previously obtained by Tonry et al. (2000) and implies that the GA mass estimate by Tonry et al. may be too high and/or that the Centaurus cluster falls into the GA almost perpendicularly to the line of sight.

5. We cannot place lower limits on the Centaurus cluster depth from our data, as the measured distance scatter of our sample $(8.1 \mathrm{Mpc})$ is almost equal to the mean single measurement uncertainty $(7.3 \mathrm{Mpc})$. We can place an upper limit of $\pm 10 \mathrm{Mpc}$ radial depth, corresponding to a five times larger radial than tangential extension.

6. We find no significant anticorrelation between redshift and distance for our data. Such a pattern would be expected in case of a backside infall into the Great Attractor. Our number counts are too low to make more definite statements, especially given the large distance uncertainty of our data.

We conclude that the deep and high resolution SBF-measurements presented here prove that the SBF-method 
allows a precise measurement of cluster distances with ground based imaging out to $40 \mathrm{Mpc}$ and beyond. The 2 highest $\mathrm{S} / \mathrm{N}$ measurements for NGC 4696 and NGC 4709 show that distance accuracies better than $10 \%$ can be achieved easily if the sampling area for SBF measurement is large enough. We have shown that it is essential to obtain sufficiently high S/N SBF data such that biases like the selection effect in distance determinations at the sensitivity limit of Tonry's SBF survey (Tonry et al. 1997) do not occur. To better investigate the structure of the Centaurus cluster with respect to its possible filamentary form and its relation to the Great Attractor, a deep and wide field survey of the entire cluster region would be needed.

Acknowledgements. The authors wish to thank the referee Dr. H. Jerjen for his very valuable comments which helped a great deal to improve the paper. SM was supported by DAAD Ph.D. grant Kennziffer D/01/35298. The authors would like to thank the ESO user support group and the ESO science operation for having carried out the programme in service mode.

\section{References}

Aaronson, M., Bothun, G. D. Mould, J., et al. 1986, ApJ, 302, 536

Aaronson, M., Bothun, G. D., Cornell, M. E., et al. 1989, ApJ, 338, 654

Bennett, C. L., Halpern, M., Hinshaw, G., et al. 2003, ApJ, submitted [astro-ph/0302207]

Blakeslee, J. P., Lucey, J. R., Tonry, J. L. et al. 2002, MNRAS, 330, 443

Bothun, G. D., Impey, C. D., \& Malin, D. F. 1991, ApJ, 376, 404

Churazov, E., Gilfanov, M., Forman, W., \& Jones, C. 1999, ApJ, 520, 105

Colberg, J. M., White, S. D. M., Jenkins, A., \& Pearce, F. R. 1999, MNRAS, 308, 593

Djorkovski, S., \& Davis, M. 1987, ApJ, 313, 59

Dressler, A., Faber, S. M., Burstein, D., et al. 1987, ApJ, 313, L37

Dressler, A. 1993, in Cosmic Velocity Fields, Proc. of the 9th IAP Astrophysics Meet., ed. F. R. Bouchet, \& M. Lachièze-Rey (Gif-sur-Yvette: Éditions Frontières), 9

Dressler, A., \& Faber, S. M. 1990, ApJ, 354, 13
Furusho, T., Yamasaki, N. Y., Ohashi, T., et al. 2001, PASJ, 53, 421 Gehrels, N. 1986, ApJ, 303, 336

Hilker, M., Mieske, S., \& Infante, L. 2003, A\&A, 397, L9

Jensen, J. B., Tonry, J. L., \& Luppino, G. A. 1998, ApJ, 505, 111

Jerjen, H., \& Dressler, A. 1997, A\&AS, 124, 1

Jerjen, H., Freeman, K. C., \& Binggeli, B. 1998, AJ, 116, 2873

Jerjen, H., Freeman, K. C., \& Binggeli, B. 2000, AJ, 119, 166

Jerjen, H., Rekola, R., Takalo, L., Coleman, M., \& Valtonen, M. 2001, A\&A, 380, 90

Jerjen, H. 2003, A\&A, 398, 63

Kissler-Patig, M., Kohle, S., Hilker, M., et al. 1997, A\&A, 319, 470

Kundu, A., \& Whitmore, B. C. 2001, AJ, 121, 2950

Lee, M., \& Geisler, D. 1997, IAU Symp. 186, abstract 62, http://www. ifa.hawaii.edu/iau-s186/abstracts/html/ 062P.html

Liu, M. C., Charlot, S., \& Graham, J. 2000, ApJ, 543, 644

Lucey, J. R., Dickens, R. J., \& Dawe, J. A. 1980, Nature, 285, 305

Lucey, J. R., Currie, M. J., \& Dickens, R. J. 1986, MNRAS, 221, 453

Lynden-Bell, D., Faber, S. M., Burstein, D., et al. 1988, ApJ, 326, 19

Malmquist, K. G. 1920, Medd. Lund. Astron. Obs., Ser. 2, No. 22

Mathewson, D. S., Ford, V. L., \& Buckhorn, M. 1992, ApJ, 389, L5

Mieske, S., Hilker, M., \& Infante, L. 2003, A\&A, 403, 43 (MieskeI)

Miller, B. W., Lotz, J., Ferguson, H. C., Stiavelli, M., \& Whitmore, B. C. 1998, ApJ, 508, L133

Schlegel, D. J., Finkbeiner, D. P., \& Davis, M. 1998, ApJ, 500, 525

Schechter, P. L. 1980, AJ, 85, 801

Shaya, E. J. 1984, ApJ, 280, 470

Spergel, D. N., Verde, L., Peiris, H. V., et al. 2003, ApJ, submitted [astro-ph/0302209]

Stein, P., Jerjen, H., \& Federspiel, M. 1997, A\&A, 327, 952

Tammann, G. A., \& Sandage, A. 1985, ApJ, 294, 81

Tonry, J. L., \& Davis, M. 1981, ApJ, 246, 680

Tonry, J. L., \& Schneider, D. P. 1988, AJ, 96, 807

Tonry, J. L., Blakeslee, J. P., Ajhar, E. A., \& Dressler, A. 1997, ApJ, 475, 399 (SBF I)

Tonry, J. L., Blakeslee, J. P., Ajhar, E. A., \& Dressler, A. 2000, ApJ, 530, 625 (SBF II)

Tonry, J. L., Dressler, A., Blakeslee, J. P., et al. 2001, ApJ, 546, 681 (SBF IV)

Worthey, G. 1994, ApJS, 95, 107

Yahil, A., Sandage, A., \& Tammann, G. A. 1980, in Lecture Notes for the 1979 Les Houches Summer School 